\title{
Metric measure spaces supporting Gagliardo-Nirenberg inequalities: volume non-collapsing and rigidities
}

\author{
Alexandru Kristály ${ }^{1,2}$
}

Received: 22 February 2016 / Accepted: 23 August 2016

(C) Springer-Verlag Berlin Heidelberg 2016

\begin{abstract}
Let $(M, \mathrm{~d}, \mathrm{~m})$ be a metric measure space which satisfies the Lott-Sturm-Villani curvature-dimension condition $\operatorname{CD}(K, n)$ for some $K \geq 0$ and $n \geq 2$, and a lower $n$-density assumption at some point of $M$. We prove that if $(M, \mathrm{~d}, \mathrm{~m})$ supports the Gagliardo-Nirenberg inequality or any of its limit cases $\left(L^{p}\right.$-logarithmic Sobolev inequality or Faber-Krahn-type inequality), then a global non-collapsing n-dimensional volume growth holds, i.e., there exists a universal constant $C_{0}>0$ such that $\mathrm{m}\left(B_{x}(\rho)\right) \geq C_{0} \rho^{n}$ for all $x \in M$ and $\rho \geq 0$, where $B_{x}(\rho)=\{y \in M: \mathrm{d}(x, y)<\rho\}$. Due to the quantitative character of the volume growth estimate, we establish several rigidity results on Riemannian manifolds with non-negative Ricci curvature supporting Gagliardo-Nirenberg inequalities by exploring a quantitative Perelman-type homotopy construction developed by Munn (J Geom Anal 20(3):723-750, 2010). Further rigidity results are also presented on some reversible Finsler manifolds.
\end{abstract}

Mathematics Subject Classification Primary 53C23; Secondary 35R06 · 53C60

\section{Contents}

1 Introduction

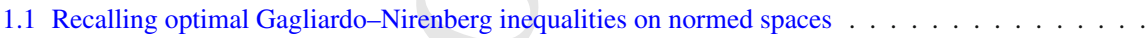

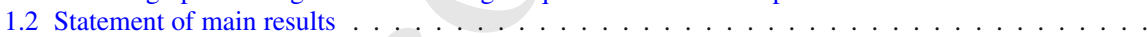

1.2.1 Volume non-collapsing on metric measure spaces . . . . . . . . . . . . . . . .

1.2.2 Applications: rigidity results in smooth settings

2 Volume non-collapsing via Gagliardo-Nirenberg inequalities

2.1 Cases $\alpha>1 \& 0<\alpha<1$ : usual Gagliardo-Nirenberg inequalities

Communicated by L. Ambrosio.

Alexandru Kristály

alex.kristaly@econ.ubbcluj.ro; alexandrukristaly@yahoo.com

1 Department of Economics, Babeş-Bolyai University, 400591 Cluj-Napoca, Romania

2 Romania and Institute of Applied Mathematics, Óbuda University, Budapest 1034, Hungary 
2.2 Limit case I $(\alpha \rightarrow 1): L^{p}$-logarithmic Sobolev inequality $\ldots \ldots \ldots \ldots \ldots \ldots$

2.3 Limit case II $(\alpha \rightarrow 0)$ : Faber-Krahn-type inequality . . . . . . . . . . . . . . . .

3 Rigidity results in smooth settings . . . . . . . . . . . . . . . . . .

3.1 Gagliardo-Nirenberg inequalities on Riemannian manifolds with $\operatorname{Ricci} \geq 0 \ldots \ldots \ldots \ldots$

3.2 Gagliardo-Nirenberg inequalities on Finsler manifolds with $n$-Ricci $\geq 0 \ldots \ldots \ldots \ldots$

References .

\section{Introduction}

An important role in the theory of geometric functional inequalities is played by the Gagliardo-Nirenberg interpolation inequality and its limit cases. The present paper is devoted to the study of Gagliardo-Nirenberg inequalities on metric measure spaces; to be more precise, we shall

(a) establish quantitative volume non-collapsing properties of metric measure spaces satisfying the Lott-Sturm-Villani curvature-dimension condition $\mathrm{CD}(K, n)$ for some $K \geq 0$ and $n \geq 2$, in the presence of a Gagliardo-Nirenberg inequality or one of its limit cases ( $L^{p}$-logarithmic Sobolev inequality or Faber-Krahn-type inequality);

(b) provide rigidity results in the framework of Riemannian and Finsler manifolds with non-negative Ricci curvature which support (almost)optimal Gagliardo-Nirenberg inequalities by using the volume non-collapsing property from (a) and a quantitative homotopy construction due to Munn [17] and Perelman [22].

In Sect. 1.1, we recall the optimal Gagliardo-Nirenberg inequalities on normed spaces which play a comparison role in our investigations; in Sect. 1.2, we present the main results of the paper.

\subsection{Recalling optimal Gagliardo-Nirenberg inequalities on normed spaces}

The optimal Gagliardo-Nirenberg inequality in the Euclidean case has been obtained by Del Pino and Dolbeault [7] for a certain range of parameters by using symmetrization arguments. By using mass transportation argument, Cordero-Erausquin et al. [6] extended the results from [7] to prove optimal Gagliardo-Nirenberg inequalities on arbitrary normed spaces. In the sequel, we recall the main theorems from [6] and some related results.

Let $\|\cdot\|$ be an arbitrary norm on $\mathbb{R}^{n}$; without loss of generality, we may assume that the Lebesgue measure of the unit ball in $\left(\mathbb{R}^{n},\|\cdot\|\right)$ is the volume of the $n$-dimensional Euclidean unit ball $\omega_{n}=\pi^{\frac{n}{2}} \Gamma\left(\frac{n}{2}+1\right)^{-1}$. The dual norm $\|\cdot\|_{*}$ of $\|\cdot\|$ is given by $\|x\|_{*}=\sup _{\|y\| \leq 1} x \cdot y$ where '.' is the Euclidean inner product. Let $p \in[1, n)$ and $L^{p}\left(\mathbb{R}^{n}\right)$ be the Lebesgue space of order $p$. As usual, we consider the Sobolev spaces

$$
\dot{W}^{1, p}\left(\mathbb{R}^{n}\right)=\left\{u \in L^{p^{\star}}\left(\mathbb{R}^{n}\right): \nabla u \in L^{p}\left(\mathbb{R}^{n}\right)\right\}
$$

and

$$
W^{1, p}\left(\mathbb{R}^{n}\right)=\left\{u \in L^{p}\left(\mathbb{R}^{n}\right): \nabla u \in L^{p}\left(\mathbb{R}^{n}\right)\right\},
$$

where $p^{\star}=\frac{p n}{n-p}$ and $\nabla$ is the gradient operator. On account of the Finslerian duality (see also Sect. 3.2), if $u \in \dot{W}^{1, p}\left(\mathbb{R}^{n}\right)$, the norm of $\nabla u$ is defined by

$$
\|\nabla u\|_{L^{p}}=\left(\int_{\mathbb{R}^{n}}\|\nabla u(x)\|_{*}^{p} d x\right)^{1 / p},
$$


where $d x$ is the Lebesgue measure on $\mathbb{R}^{n}$.

Fix $n \geq 2, p \in(1, n)$ and $\alpha \in\left(0, \frac{n}{n-p}\right] \backslash\{1\}$; for every $\lambda>0$, let

$$
h_{\alpha, p}^{\lambda}(x)=\left(\lambda+(\alpha-1)\|x\|^{p^{\prime}}\right)_{+}^{\frac{1}{1-\alpha}}, \quad x \in \mathbb{R}^{n}, 1
$$

where $p^{\prime}=\frac{p}{p-1}$ is the conjugate to $p$, and $r_{+}=\max \{0, r\}$ for $r \in \mathbb{R}$. The following optimal Gagliardo-Nirenberg inequalities are known on normed spaces:

Theorem A. (see [6, Theorem 4]) Let $n \geq 2, p \in(1, n)$ and $\|\cdot\|$ be an arbitrary norm on $\mathbb{R}^{n}$.

- If $1<\alpha \leq \frac{n}{n-p}$, then

$$
\|u\|_{L^{\alpha p}} \leq \mathcal{G}_{\alpha, p, n}\|\nabla u\|_{L^{p}}^{\theta}\|u\|_{L^{\alpha(p-1)+1}}^{1-\theta}, \quad \forall u \in \dot{W}^{1, p}\left(\mathbb{R}^{n}\right),
$$

where

$$
\theta=\frac{p^{\star}(\alpha-1)}{\alpha p\left(p^{\star}-\alpha p+\alpha-1\right)}
$$

and the best constant

$$
\mathcal{G}_{\alpha, p, n}=\left(\frac{\alpha-1}{p^{\prime}}\right)^{\theta} \frac{\left(\frac{p^{\prime}}{n}\right)^{\frac{\theta}{p}+\frac{\theta}{n}}\left(\frac{\alpha(p-1)+1}{\alpha-1}-\frac{n}{p^{\prime}}\right)^{\frac{1}{\alpha p}}\left(\frac{\alpha(p-1)+1}{\alpha-1}\right)^{\frac{\theta}{p}-\frac{1}{\alpha p}}}{\left(\omega_{n} \mathrm{~B}\left(\frac{\alpha(p-1)+1}{\alpha-1}-\frac{n}{p^{\prime}}, \frac{n}{p^{\prime}}\right)\right)^{\frac{\theta}{n}}}
$$

is achieved by the family of functions $h_{\alpha, p}^{\lambda}, \lambda>0$;

- If $0<\alpha<1$, then

$$
\|u\|_{L^{\alpha(p-1)+1}} \leq \mathcal{N}_{\alpha, p, n}\|\nabla u\|_{L^{p}}^{\gamma}\|u\|_{L^{\alpha p}}^{1-\gamma}, \quad \forall u \in \dot{W}^{1, p}\left(\mathbb{R}^{n}\right),
$$

where

$$
\gamma=\frac{p^{\star}(1-\alpha)}{\left(p^{\star}-\alpha p\right)(\alpha p+1-\alpha)},
$$

and the best constant

$$
\mathcal{N}_{\alpha, p, n}=\left(\frac{1-\alpha}{p^{\prime}}\right)^{\gamma} \frac{\left(\frac{p^{\prime}}{n}\right)^{\frac{\gamma}{p}+\frac{\gamma}{n}}\left(\frac{\alpha(p-1)+1}{1-\alpha}+\frac{n}{p^{\prime}}\right)^{\frac{\gamma}{p}-\frac{1}{\alpha(p-1)+1}}\left(\frac{\alpha(p-1)+1}{1-\alpha}\right)^{\frac{1}{\alpha(p-1)+1}}}{\left(\omega_{n} \mathrm{~B}\left(\frac{\alpha(p-1)+1}{1-\alpha}, \frac{n}{p^{\prime}}\right)\right)^{\frac{\gamma}{n}}}
$$

is achieved by the family of functions $h_{\alpha, p}^{\lambda}, \lambda>0$.

\section{Hereafter, $\mathrm{B}(\cdot, \cdot)$ is the Euler beta-function.}

The borderline case $\alpha=\frac{n}{n-p}$ (thus $\theta=1$ ) reduces to the optimal Sobolev inequality, see Aubin [3] and Talenti [26] in the Euclidean case, and Alvino et al. [1] for normed spaces. Furthermore, inequalities (1.1) and (1.3) degenerate to the optimal $L^{p}$-logarithmic Sobolev inequality whenever $\alpha \rightarrow 1$ (called also as the entropy-energy inequality involving the Shannon entropy), while (1.3) reduces to a Faber-Krahn-type inequality whenever $\alpha \rightarrow 0$, respectively. More precisely, one has

1 The function $h_{\alpha, p}^{\lambda}$ is positive everywhere for $\alpha>1$ while $h_{\alpha, p}^{\lambda}$ has always a compact support for $\alpha<1$. 
Theorem B. Let $n \geq 2, p \in(1, n)$ and $\|\cdot\|$ be an arbitrary norm on $\mathbb{R}^{n}$.

- Limit case $\mathbf{I}(\alpha \rightarrow 1)$ (see [9, Theorem 1.1] $\left.]^{2}\right)$ : One has

$$
\begin{aligned}
& \text { Ent }_{d x}\left(|u|^{p}\right)=\int_{\mathbb{R}^{n}}|u|^{p} \log |u|^{p} d x \leq \frac{n}{p} \log \left(\mathcal{L}_{p, n}\|\nabla u\|_{L^{p}}^{p}\right), \\
& \forall u \in W^{1, p}\left(\mathbb{R}^{n}\right),\|u\|_{L^{p}}=1,
\end{aligned}
$$

where the best constant

$$
\mathcal{L}_{p, n}=\frac{p}{n}\left(\frac{p-1}{e}\right)^{p-1}\left(\omega_{n} \Gamma\left(\frac{n}{p^{\prime}}+1\right)\right)^{-\frac{p}{n}}
$$

is achieved by the family of functions

$$
l_{p}^{\lambda}(x)=\lambda^{\frac{n}{p p^{\prime}}} \omega_{n}^{-\frac{1}{p}} \Gamma\left(\frac{n}{p^{\prime}}+1\right)^{-\frac{1}{p}} e^{-\frac{\lambda}{p}\|x\|^{p^{\prime}}}, \quad \lambda>0 ;
$$

- Limit case II ( $\alpha \rightarrow 0)$ (see [6, p. 320]): One has

$$
\|u\|_{L^{1}} \leq \mathcal{F}_{p, n}\|\nabla u\|_{L^{p}}|\operatorname{supp}(u)|^{1-\frac{1}{p^{\star}}}, \quad \forall u \in \dot{W}^{1, p}\left(\mathbb{R}^{n}\right)
$$

and the best constant

$$
\mathcal{F}_{p, n}=\lim _{\alpha \rightarrow 0} \mathcal{N}_{\alpha, p, n}=n^{-\frac{1}{p}} \omega_{n}^{-\frac{1}{n}}\left(p^{\prime}+n\right)^{-\frac{1}{p^{\prime}}}
$$

is achieved by the family of functions

$$
f_{p}^{\lambda}(x)=\lim _{\alpha \rightarrow 0} h_{\alpha, p}^{\lambda}(x)=\left(\lambda-\|x\|^{p^{\prime}}\right)_{+}, \quad x \in \mathbb{R}^{n},
$$

where $\operatorname{supp}(u)$ stands for the support of $u$ and $|\operatorname{supp}(u)|$ is its Lebesgue measure.

\subsection{Statement of main results}

As we already pointed out, the primordial purpose of the present paper is to establish fine topological properties of metric measure spaces curved in the sense of Lott-Sturm-Villani which support Gagliardo-Nirenberg-type inequalities. In fact, the metric spaces we are working on are supposed to satisfy the curvature-dimension condition $\operatorname{CD}(K, n)$ for some $K \geq 0$ and $n \geq 2$, introduced by Lott and Villani [15] and Sturm [24,25]; see Sect. 2 for its formal definition.

\subsubsection{Volume non-collapsing on metric measure spaces}

Let $(M, \mathrm{~d}, \mathrm{~m})$ be a metric measure space (with a strictly positive Borel measure $\mathrm{m}$ ) and $\operatorname{Lip}_{0}(M)$ be the space of Lipschitz functions with compact support on $M$. For $u \in \operatorname{Lip}_{0}(M)$, let

$$
|\nabla u|_{\mathrm{d}}(x):=\limsup _{y \rightarrow x} \frac{|u(y)-u(x)|}{\mathrm{d}(x, y)}, \quad x \in M .
$$

Note that $x \mapsto|\nabla u|_{\mathrm{d}}(x)$ is Borel measurable on $M$ for $u \in \operatorname{Lip}_{0}(M)$.

2 Gentil [9] proved an optimal $L^{p}$-logarithmic Sobolev inequality for even, $q$-homogeneous $(q>1)$, strictly convex functions $C: \mathbb{R}^{n} \rightarrow[0, \infty)$. In our case, $C(x)=\frac{\|x\|^{p^{\prime}}}{p^{\prime}}$.

\section{Springer}


As before, let $n \geq 2$ be an integer, $p \in(1, n)$ and $\alpha \in\left(0, \frac{n}{n-p}\right] \backslash\{1\}$. Throughout this section we assume that the lower $n$-density of the measure $m$ at a point $x_{0} \in M$ is unitary, i.e.,

$$
\text { (D) } \mathrm{x}_{\mathrm{x}_{0}}^{\mathrm{n}}: \liminf _{\rho \rightarrow 0} \frac{\mathrm{m}\left(B_{x_{0}}(\rho)\right)}{\omega_{n} \rho^{n}}=1,
$$

where $B_{x}(r)=\{y \in M: \mathrm{d}(x, y)<r\}$.

Throughout the whole paper, we shall keep the notations from Theorems A and B [i.e., the four best constants from the Gagliardo-Nirenberg inequalities on normed spaces and the numbers $\theta$ and $\gamma$ from (1.2) and (1.4), respectively]; the Lebesgue spaces $L^{p}$ are defined on the measure space $(M, \mathrm{~m})$. We now are the position to state our quantitative, globally non-collapsing volume growth results:

Theorem 1.1 (Gagliardo-Nirenberg inequalities) Let $(M, \mathrm{~d}, \mathrm{~m})$ be a proper metric measure space which satisfies the curvature-dimension condition $\operatorname{CD}(K, n)$ for some $K \geq 0$ and $n \geq 2$. Let $p \in(1, n)$ and assume that $(\mathbf{D})_{x_{0}}^{n}$ holds for some $x_{0} \in M$. Then the following statements hold:

(i) If $1<\alpha \leq \frac{n}{n-p}$ and the inequality

$$
\|u\|_{L^{\alpha p}} \leq \mathcal{C}\left\||\nabla u|_{\mathrm{d}}\right\|_{L^{p}}^{\theta}\|u\|_{L^{\alpha(p-1)+1}}^{1-\theta}, \quad \forall u \in \operatorname{Lip}_{0}(M)
$$

$(\mathbf{G N 1})_{\mathcal{C}}^{\alpha, p}$

holds for some $\mathcal{C} \geq \mathcal{G}_{\alpha, p, n}$, then $K=0$ and

$$
\mathrm{m}\left(B_{x}(\rho)\right) \geq\left(\frac{\mathcal{G}_{\alpha, p, n}}{\mathcal{C}}\right)^{\frac{n}{\theta}} \omega_{n} \rho^{n} \quad \text { forall } x \in M \text { and } \rho \geq 0 .
$$

(ii) If $0<\alpha<1$ and the inequality

$$
\|u\|_{L^{\alpha(p-1)+1}} \leq \mathcal{C}\left\||\nabla u|_{\mathrm{d}}\right\|_{L^{p}}^{\gamma}\|u\|_{L^{\alpha p}}^{1-\gamma}, \forall u \in \operatorname{Lip}_{0}(M)
$$

holds for some $\mathcal{C} \geq \mathcal{N}_{\alpha, p, n}$, then $K=0$ and

$$
\mathrm{m}\left(B_{x}(\rho)\right) \geq\left(\frac{\mathcal{N}_{\alpha, p, n}}{\mathcal{C}}\right)^{\frac{n}{\gamma}} \omega_{n} \rho^{n} \text { for all } x \in M \text { and } \rho \geq 0 .
$$

In the limit case $\alpha \rightarrow 1$, we can state

Theorem 1.2 ( $L^{p}$-logarithmic Sobolev inequality) Under the same assumptions as in Theorem 1.1, if

$$
\begin{aligned}
\text { Ent }_{d \mathrm{~m}}\left(|u|^{p}\right) & =\int_{M}|u|^{p} \log |u|^{p} d \mathrm{~m} \leq \frac{n}{p} \log \left(\mathcal{C}\left\||\nabla u|_{\mathrm{d}}\right\|_{L^{p}}^{p}\right), \quad \forall u \in \operatorname{Lip}_{0}(M), \\
\|u\|_{L^{p}}=1 & (\mathbf{L S})_{\mathcal{C}}^{p}
\end{aligned}
$$

holds for some $\mathcal{C} \geq \mathcal{L}_{p, n}$, then $K=0$ and

$$
\mathrm{m}\left(B_{x}(\rho)\right) \geq\left(\frac{\mathcal{L}_{p, n}}{\mathcal{C}}\right)^{\frac{n}{p}} \omega_{n} \rho^{n} \text { for all } x \in M \text { and } \rho \geq 0 .
$$

In the remaining limit case $\alpha \rightarrow 0$, one can prove 
Theorem 1.3 (Faber-Krahn-type inequality) Under the same assumptions as in Theorem 1.1, if

$$
\|u\|_{L^{1}} \leq \mathcal{C}\left\||\nabla u|_{\mathrm{d}}\right\|_{L^{p}} \mathrm{~m}(\operatorname{supp}(u))^{1-\frac{1}{p^{\star}}}, \quad \forall u \in \operatorname{Lip}_{0}(M)
$$

holds for some $\mathcal{C} \geq \mathcal{F}_{p, n}$, then $K=0$ and

$$
\mathrm{m}\left(B_{x}(\rho)\right) \geq\left(\frac{\mathcal{F}_{p, n}}{\mathcal{C}}\right)^{n} \omega_{n} \rho^{n} \text { for all } x \in M \text { and } \rho \geq 0 \text {. }
$$

Some remarks are in order.

Remark 1.1 (a) The proofs of Theorems 1.1-1.3 are synthetic where we shall exploit some basic features of metric measure spaces satisfying the $\operatorname{CD}(K, n)$ condition (such as generalized Bonnet-Myers and Bishop-Gromov comparison inequalities) and direct constructions. Although the lines of the proofs of these results are similar, our arguments require different technics, deeply depending on the shape of certain test functions whose profiles come from the family of extremals in normed spaces (cf. Theorems A \& B). Note that instead of the $\mathrm{CD}(K, n)$ condition it is enough to consider the slightly weaker measure contraction property $\operatorname{MCP}(K, n)$, see Ohta [20].

(b) The case $p=2$ and $\alpha=\frac{n}{n-2}(n \geq 3)$ is contained in Kristály and Ohta [12], where the authors studied Caffarelli-Kohn-Nirenberg inequalities on metric measure spaces. We notice that the roots of Theorem 1.1 (i) on Riemannian manifolds with non-negative Ricci curvature can be found in do Carmo and Xia [8], Ledoux [13] and Xia [28].

(c) The generalized Bishop-Gromov inequality and density assumption $(\mathbf{D})_{x_{0}}^{n}$ imply $\mathrm{m}\left(B_{x_{0}}(\rho)\right) \leq \omega_{n} \rho^{n}$ for all $\rho \geq 0$. In particular, the latter inequality and the conclusions of Theorems $1.1-1.3$ imply the Ahlfors $n$-regularity at the point $x_{0}$; therefore, the Hausdorff dimension of $(M, \mathrm{~d})$ is precisely $n$.

(d) (D) $x_{0}$ clearly holds for every point $x_{0}$ on $n$-dimensional Riemannian and Finsler manifolds endowed with the canonical Busemann-Hausdorff measure.

\subsubsection{Applications: rigidity results in smooth settings}

Having fine volume growth estimates in Theorems 1.1-1.3, important rigidity results can be deduced in the context of Riemannian and Finsler manifolds supporting GagliardoNirenberg-type inequalities.

In order to state such results, let $(M, g)$ be an $n$-dimensional complete Riemannian manifold with non-negative Ricci curvature $(n \geq 2)$ endowed with its canonical volume form $d v_{g}$. Let $\alpha_{M P}(k, n) \in(0,1]$ be the so-called Munn-Perelman constant for every $k=1, \ldots, n$, see Munn [17]. In fact, based on the double induction argument of Perelman [22], Munn determined explicit lower bounds for the volume growth in terms of the constant $\alpha_{M P}(k, n)$ which guarantee the triviality of the $k$-th homotopy group $\pi_{k}(M)$ of $(M, g)$; see details in Sect. 3.

For sake of simplicity, we restrict here our attention to the $L^{p}$-logarithmic Sobolev inequality $(\mathbf{L S})_{\mathcal{C}}^{p}$ on $(M, g)$ by proving that once $\mathcal{C}>0$ is closer and closer to the optimal Euclidean constant $\mathcal{L}_{p, n}$, the manifold $(M, g)$ approaches topologically more and more to the Euclidean space $\mathbb{R}^{n}$.

Theorem 1.4 Let $(M, g)$ be an $n$-dimensional complete Riemannian manifold with nonnegative Ricci curvature $(n \geq 2)$ and assume the $L^{p}$-logarithmic Sobolev inequality $(\mathbf{L S})_{\mathcal{C}}^{p}$ holds on $(M, g)$ for some $p \in(1, n)$ and $\mathcal{C}>0$. Then the following assertions hold: 
(i) $\mathcal{C} \geq \mathcal{L}_{p, n}$;

(ii) The order of the fundamental group $\pi_{1}(M)$ is bounded above by $\left(\frac{\mathcal{C}}{\mathcal{L}_{p, n}}\right)^{\frac{n}{p}}$;

(iii) If $<\alpha_{M P}\left(k_{0}, n\right)^{-\frac{p}{n}} \mathcal{L}_{p, n}$ for some $k_{0} \in\{1, \ldots, n\}$ then $\pi_{1}(M)=\cdots=\pi_{k_{0}}(M)=0$;

(iv) If $\mathcal{C}<\alpha_{M P}(n, n)^{-\frac{p}{n}} \mathcal{L}_{p, n}$ then $M$ is contractible;

(v) $\mathcal{C}=\mathcal{L}_{p, n}$ if and only if $(M, g)$ is isometric to the Euclidean space $\mathbb{R}^{n}$.

Remark 1.2 (a) Theorem 1.4 (v) answers an open question of Xia [29] for generic $p \in$ $(1, n)$. For $p=2$ the latter equivalence is well known by using sharp analytic estimates for the heat kernel on complete Riemannian manifolds with non-negative Ricci curvature; see Bakry et al. [4], Ni [18], and Li [14]. Details are presented in Sect. 3.1 (see Remark 3.1).

(b) The conclusion $\mathcal{C} \geq \mathcal{L}_{p, n}$ in Theorem 1.4 (i) is in a perfect concordance with the assumption of Theorem 1.2. Analogous statements hold for the other Gagliardo-Nirenberg inequalities.

(c) Similar results to Theorem 1.4 can be stated also for Gagliardo-Nirenberg inequalities $(\mathbf{G N 1})_{\mathcal{C}}$ and $(\mathbf{G N 2})_{\mathcal{C}}$, and Faber-Krahn inequality $(\mathbf{F K})_{\mathcal{C}}$ with trivial modifications. In particular, we have:

Corollary 1.1 (Optimality vs. flatness) Let $(M, g)$ be an $n(\geq 2)$-dimensional complete Riemannian manifold with non-negative Ricci curvature. The following statements are equivalent:

(i) (GN1) ${ }_{\mathcal{G}_{\alpha, p, n}}^{\alpha, p}$ holds on $(M, g)$ for some $p \in(1, n)$ and $\alpha \in\left(1, \frac{n}{n-p}\right]$;

(ii) (GN2) ${ }_{\mathcal{N}_{\alpha, p, n}^{\alpha, p}}^{\alpha, p l d s}$ on $(M, g)$ for some $p \in(1, n)$ and $\alpha \in(0,1)$;

(iii) $(\mathbf{L S})_{\mathcal{L}_{p, n}}^{p}$ holds on $(M, g)$ for some $p \in(1, n)$;

(iv) $(\mathbf{F K})_{\mathcal{F}_{p, n}}^{p}$ holds on $(M, g)$ for some $p \in(1, n)$;

(v) $(M, g)$ is isometric to the Euclidean space $\mathbb{R}^{n}$.

Remark 1.3 (a) The equivalence (i) $\Leftrightarrow(\mathrm{v})$ in Corollary 1.1 is precisely the main result of Xia [28].

(b) A similar rigidity result to Corollary 1.1 can be stated on reversible Finsler manifolds endowed with the natural Busemann-Hausdoff measure $d V_{F}$ of $(M, F)$; roughly speaking, we can replace the notions 'Riemannian' and 'Euclidean' in Corollary 1.1 by the notions 'Berwald' and 'Minkowski', respectively (see Theorem 3.2). The latter notions will be introduced in Sect. 3.2.

Notations. When no confusion arises, $\|\cdot\|_{L^{p}}$ abbreviates: (a) $\|\cdot\|_{L^{p}(M, d \mathrm{~m})}$ on the metric measure space $(M, \mathrm{~d}, \mathrm{~m}) ;(\mathrm{b})\|\cdot\|_{L^{p}\left(M, d v_{g}\right)}$ on the Riemannian manifold $(M, g)$ where $d v_{g}$ stands for the canonical Riemannian measure on $(M, g)$; (c) $\|\cdot\|_{L^{p}\left(M, d V_{F}\right)}$ on the Finsler manifold $(M, F)$ where $d V_{F}$ denotes the Busemann-Hausdoff measure on $(M, F)$; and (d) $\|\cdot\|_{L^{p}\left(\mathbb{R}^{n}, d x\right)}$ on the Euclidean/normed space $\mathbb{R}^{n}$ where $d x$ is the usual Lebesgue measure, respectively. When $A$ is not the whole space we are working on, we shall use the notation $\|u\|_{L^{p}(A)}$ for the $L^{p}$-norm of the function $u: A \rightarrow \mathbb{R}$.

\section{Volume non-collapsing via Gagliardo-Nirenberg inequalities}

Before the presentation of the proofs of Theorems 1.1-1.3, we recall for completeness some notions and results from Lott and Villani [15] and Sturm [24,25], which are indispensable in our arguments. 
Let $(M, \mathrm{~d}, \mathrm{~m})$ be a metric measure space, i.e., $(M, \mathrm{~d})$ is a complete separable metric space and $\mathrm{m}$ is a locally finite measure on $M$ endowed with its Borel $\sigma$-algebra. In the sequel, we assume that the measure $\mathrm{m}$ on $M$ is strictly positive, i.e., $\operatorname{supp}[\mathrm{m}]=M$. As usual, $\mathcal{P}_{2}(M, \mathrm{~d})$ is the $L^{2}$-Wasserstein space of probability measures on $M$, while $\mathcal{P}_{2}(M, \mathrm{~d}, \mathrm{~m})$ will denote the subspace of $\mathrm{m}$-absolutely continuous measures. $(M, \mathrm{~d}, \mathrm{~m})$ is said to be proper if every bounded and closed subset of $M$ is compact.

For a given number $N \geq 1$, the Rényi entropy functional $S_{N}(\cdot \mid \mathrm{m}): \mathcal{P}_{2}(M, \mathrm{~d}) \rightarrow \mathbb{R}$ with respect to the measure $\mathrm{m}$ is defined by $S_{N}(\mu \mid \mathrm{m})=-\int_{M} \rho^{-\frac{1}{N}} d \mu, \rho$ being the density of $\mu^{c}$ in $\mu=\mu^{c}+\mu^{s}=\rho \mathrm{m}+\mu^{s}$, where $\mu^{c}$ and $\mu^{s}$ represent the absolutely continuous and singular parts of $\mu \in \mathcal{P}_{2}(M, \mathrm{~d})$, respectively.

Let $K, N \in \mathbb{R}$ be two numbers with $K \geq 0$ and $N \geq 1$. For every $t \in[0,1]$ and $s \geq 0$, let

$$
\tau_{K, N}^{(t)}(s)= \begin{cases}+\infty, & \text { if } K s^{2} \geq(N-1) \pi^{2} \\ t^{\frac{1}{N}}\left(\sin \left(\sqrt{\frac{K}{N-1}} t s\right) / \sin \left(\sqrt{\frac{K}{N-1}} s\right)\right)^{1-\frac{1}{N}}, & \text { if } 0<K s^{2}<(N-1) \pi^{2} \\ t, & \text { if } K s^{2}=0 .\end{cases}
$$

We say that $(M, \mathrm{~d}, \mathrm{~m})$ satisfies the curvature-dimension condition $\operatorname{CD}(K, N)$ if for each $\mu_{0}, \mu_{1} \in \mathcal{P}_{2}(M, \mathrm{~d}, \mathrm{~m})$ there exists an optimal coupling $\gamma$ of $\mu_{0}, \mu_{1}$ and a geodesic $\Gamma$ : $[0,1] \rightarrow \mathcal{P}_{2}(M, \mathrm{~d}, \mathrm{~m})$ joining $\mu_{0}$ and $\mu_{1}$ such that

$$
S_{N^{\prime}}(\Gamma(t) \mid \mathrm{m}) \leq-\int_{M \times M}\left[\tau_{K, N^{\prime}}^{(1-t)}\left(\mathrm{d}\left(x_{0}, x_{1}\right)\right) \rho_{0}^{-\frac{1}{N^{\prime}}}\left(x_{0}\right)+\tau_{K, N^{\prime}}^{(t)}\left(\mathrm{d}\left(x_{0}, x_{1}\right)\right) \rho_{1}^{-\frac{1}{N^{\prime}}}\left(x_{1}\right)\right] d \gamma\left(x_{0}, x_{1}\right)
$$

for every $t \in[0,1]$ and $N^{\prime} \geq N$, where $\rho_{0}$ and $\rho_{1}$ are the densities of $\mu_{0}$ and $\mu_{1}$ with respect to $\mathrm{m}$. Clearly, when $K=0$, the above inequality reduces to the the geodesic convexity of $S_{N^{\prime}}(\cdot \mid \mathrm{m})$ on the $L^{2}$-Wasserstein space $\mathcal{P}_{2}(M, \mathrm{~d}, \mathrm{~m})$.

It is well known that $\operatorname{CD}(K, n)$ holds on a complete Riemannian manifold $(M, g)$ endowed with the Riemannian volume element $d v_{g}$ if and only if its Ricci curvature $\geq K$ and $\operatorname{dim}(M) \leq n$.

Let $B_{x}(r)=\{y \in M: \mathrm{d}(x, y)<r\}$. In the sequel we shall exploit properties which are resumed in the following results.

Theorem 2.1 (see [25]) Let $(M, \mathrm{~d}, \mathrm{~m})$ be a metric measure space with strictly positive measure $\mathrm{m}$ satisfying the curvature-dimension condition $\operatorname{CD}(K, N)$ for some $K \geq 0$ and $N>1$. Then every bounded set $S \subset M$ has finite $\mathrm{m}$-measure and the metric spheres $\partial B_{x}(r)$ have zero $\mathrm{m}$-measures. Moreover, one has:

(i) [Generalized Bonnet-Myers theorem] If $K>0$, then $M=\operatorname{supp}[\mathrm{m}]$ is compact and has diameter less than or equal to $\sqrt{\frac{N-1}{K}} \pi$.

(ii) [Generalized Bishop-Gromov inequality] If $K=0$, then for every $R>r>0$ and $x \in M$,

$$
\frac{\mathrm{m}\left(B_{x}(r)\right)}{r^{N}} \geq \frac{\mathrm{m}\left(B_{x}(R)\right)}{R^{N}} .
$$

Lemma 2.1 Let $(M, \mathrm{~d}, \mathrm{~m})$ be a metric measure space which satisfies the curvaturedimension condition $\mathrm{CD}(0, n)$ for some $n \geq 2$. If

$$
\ell_{\infty}^{x_{0}}:=\limsup _{\rho \rightarrow \infty} \frac{\mathrm{m}\left(B_{x_{0}}(\rho)\right)}{\omega_{n} \rho^{n}} \geq a
$$

\section{Springer}


for some $x_{0} \in M$ and $a>0$, then

$$
\mathrm{m}\left(B_{x}(\rho)\right) \geq a \omega_{n} \rho^{n}, \quad \forall x \in M, \rho \geq 0 .
$$

Proof Let us fix $x \in M$ and $\rho>0$; then we have

$$
\begin{array}{rlr}
\frac{\mathrm{m}\left(B_{x}(\rho)\right)}{\omega_{n} \rho^{n}} & \geq \limsup _{r \rightarrow \infty} \frac{\mathrm{m}\left(B_{x}(r)\right)}{\omega_{n} r^{n}} & \text { [Bishop - Gromov inequality] } \\
& \geq \limsup _{r \rightarrow \infty} \frac{\mathrm{m}\left(B_{x_{0}}\left(r-\mathrm{d}\left(x_{0}, x\right)\right)\right)}{\omega_{n} r^{n}} & {\left[B_{x}(r) \supset B_{x_{0}}\left(r-\mathrm{d}\left(x_{0}, x\right)\right)\right]} \\
& =\limsup _{r \rightarrow \infty}\left(\frac{\mathrm{m}\left(B_{x_{0}}\left(r-\mathrm{d}\left(x_{0}, x\right)\right)\right)}{\omega_{n}\left(r-\mathrm{d}\left(x_{0}, x\right)\right)^{n}} \cdot \frac{\left(r-\mathrm{d}\left(x_{0}, x\right)\right)^{n}}{r^{n}}\right) \\
& =\ell_{\infty}^{x_{0}} & \\
& \geq a, & {[\mathrm{cf} .(2.1)]}
\end{array}
$$

which concludes the proof.

We are now in the position to prove our volume non-collapsing results.

\subsection{Cases $\alpha>1 \& 0<\alpha<1$ : usual Gagliardo-Nirenberg inequalities}

In this subsection we present the proof of Theorem 1.1 by distinguishing two cases:

Proof of Theorem 1.1 (i): the case $1<\alpha \leq \frac{n}{n-p}$. In this part, we follow the line of [12]; the proof is divided into several steps. We clearly may assume that $\mathcal{C}>\mathcal{G}_{\alpha, p, n}$ in $(\mathbf{G N 1})_{\mathcal{C}}^{\alpha, p}$; indeed, if $\mathcal{C}=\mathcal{G}_{\alpha, p, n}$ we can consider the subsequent arguments for $\mathcal{C}:=\mathcal{G}_{\alpha, p, n}+\varepsilon$ with small $\varepsilon>0$ and then take $\varepsilon \rightarrow 0^{+}$.

Step $1(K=0)$. If we assume that $K>0$ then the generalized Bonnet-Myers theorem (see Theorem 2.1 (i)) implies that $M$ is compact and $\mathrm{m}(M)$ is finite. Taking the constant map $u(x)=\mathrm{m}(M)$ in $(\mathbf{G N 1})_{\mathcal{C}}^{\alpha, p}$ as a test function, one gets a contradiction. Therefore, $K=0$.

Step 2 (ODE from the optimal Euclidean Gagliardo-Nirenberg inequality I). We consider the optimal Gagliardo-Nirenberg inequality (1.1) in the particular case when the norm is precisely the Euclidean norm $|\cdot|$. After a simple rescaling, one can see that the function $x \mapsto\left(\lambda+|x|^{p^{\prime}}\right)^{\frac{1}{1-\alpha}}, \lambda>0$, is a family of extremals in (1.1); therefore, we have the following first order ODE

$$
\begin{aligned}
\left(\frac{1-\alpha}{\alpha(p-1)+1} h_{G}^{\prime}(\lambda)\right)^{\frac{1}{\alpha p}}= & \mathcal{G}_{\alpha, p, n}\left(\frac{p^{\prime}}{\alpha-1}\right)^{\theta}\left(h_{G}(\lambda)+\frac{\alpha-1}{\alpha(p-1)+1} \lambda h_{G}^{\prime}(\lambda)\right)^{\frac{\theta}{p}} \\
& h_{G}(\lambda)^{\frac{1-\theta}{\alpha(p-1)+1},}
\end{aligned}
$$

where $h_{G}:(0, \infty) \rightarrow \mathbb{R}$ is given by

$$
h_{G}(\lambda)=\int_{\mathbb{R}^{n}}\left(\lambda+|x|^{p^{\prime}}\right)^{\frac{\alpha(p-1)+1}{1-\alpha}} d x, \quad \lambda>0 .
$$

For further use, we shall represent the function $h_{G}$ in two different ways, namely

$$
\begin{aligned}
h_{G}(\lambda) & =\omega_{n} \frac{n}{p^{\prime}} \mathrm{B}\left(\frac{\alpha(p-1)+1}{\alpha-1}-\frac{n}{p^{\prime}}, \frac{n}{p^{\prime}}\right) \lambda^{\frac{\alpha(p-1)+1}{1-\alpha}+\frac{n}{p^{\prime}}} \\
& =\int_{0}^{\infty} \omega_{n} \rho^{n} f_{G}(\lambda, \rho) d \rho,
\end{aligned}
$$


where

$$
f_{G}(\lambda, \rho)=p^{\prime} \frac{\alpha(p-1)+1}{\alpha-1}\left(\lambda+\rho^{p^{\prime}}\right)^{\frac{\alpha p}{1-\alpha}} \rho^{p^{\prime}-1} .
$$

Step 3 (Differential inequality from $(\mathbf{G N 1})_{\mathcal{C}}^{\alpha, p}$ ). By the generalized Bishop-Gromov inequality (see Theorem 2.1 (ii)) and hypothesis $(\mathbf{D})_{x_{0}}^{n}$ one has that

$$
\frac{\mathrm{m}\left(B_{x_{0}}(\rho)\right)}{\omega_{n} \rho^{n}} \leq \liminf _{r \rightarrow 0} \frac{\mathrm{m}\left(B_{x_{0}}(r)\right)}{\omega_{n} r^{n}}=1, \quad \rho>0 .
$$

Inspired by the form of $h_{G}$, we consider the function $w_{G}:(0, \infty) \rightarrow \mathbb{R}$ defined by

$$
w_{G}(\lambda)=\int_{M}\left(\lambda+\mathrm{d}\left(x_{0}, x\right)^{p^{\prime}}\right)^{\frac{\alpha(p-1)+1}{1-\alpha}} d \mathrm{~m}(x), \quad \lambda>0 .
$$

By using the layer cake representation, it follows that $w_{G}$ is well-defined and of class $C^{1}$; indeed,

$$
\begin{array}{rlrl}
w_{G}(\lambda) & =\int_{0}^{\infty} \mathrm{m}\left(\left\{x \in M:\left(\lambda+\mathrm{d}\left(x_{0}, x\right)^{p^{\prime}}\right)^{\frac{\alpha(p-1)+1}{1-\alpha}}>t\right\}\right) d t \\
& =\int_{0}^{\infty} \mathrm{m}\left(B_{x_{0}}(\rho)\right) f_{G}(\lambda, \rho) d \rho & \text { [change } t=\left(\lambda+\rho^{p^{\prime}}\right)^{\frac{\alpha(p-1)+1}{1-\alpha}} \text { and see } \\
& \leq \int_{0}^{\infty} \omega_{n} \rho^{n} f_{G}(\lambda, \rho) d \rho & {[\text { see }(2.5)]} \\
& =h_{G}(\lambda)
\end{array}
$$

thus

$$
0<w_{G}(\lambda) \leq h_{G}(\lambda)<\infty, \lambda>0 .
$$

For every $\lambda>0$ and $k \in \mathbb{N}$, we consider the function $u_{\lambda, k}: M \rightarrow \mathbb{R}$ defined by

$$
u_{\lambda, k}(x)=\left(\min \left\{0, k-\mathrm{d}\left(x_{0}, x\right)\right\}+1\right)_{+}\left(\lambda+\max \left\{\mathrm{d}\left(x_{0}, x\right), k^{-1}\right\}^{p^{\prime}}\right)^{\frac{1}{1-\alpha}} .
$$

Note that since $(M, \mathrm{~d}, \mathrm{~m})$ is proper, the $\operatorname{set} \operatorname{supp}\left(u_{\lambda, k}\right)=\overline{B_{x_{0}}(k+1)}$ is compact. Consequently, $u_{\lambda, k} \in \operatorname{Lip}_{0}(M)$ for every $\lambda>0$ and $k \in \mathbb{N}$; thus we can apply these functions in $(\mathbf{G N 1})_{\mathcal{C}}^{\alpha, p}$, i.e.,

$$
\left\|u_{\lambda, k}\right\|_{L^{\alpha p}} \leq \mathcal{C}\left\|\left|\nabla u_{\lambda, k}\right|_{\mathrm{d}}\right\|_{L^{p}}^{\theta}\left\|u_{\lambda, k}\right\|_{L^{\alpha(p-1)+1}}^{1-\theta}
$$

Moreover,

$$
\lim _{k \rightarrow \infty} u_{\lambda, k}(x)=\left(\lambda+\mathrm{d}\left(x_{0}, x\right)^{p^{\prime}}\right)^{\frac{1}{1-\alpha}}=: u_{\lambda}(x) .
$$

By using the dominated convergence theorem, it turns out from the above inequality that $u_{\lambda}$ also verifies $(\mathbf{G N 1})_{\mathcal{C}}^{\alpha, p}$, i.e.,

$$
\left\|u_{\lambda}\right\|_{L^{\alpha p}} \leq \mathcal{C}\left\|\left|\nabla u_{\lambda}\right|_{\mathrm{d}}\right\|_{L^{p}}^{\theta}\left\|u_{\lambda}\right\|_{L^{\alpha(p-1)+1}}^{1-\theta} .
$$

The non-smooth chain rule gives that

$$
\left|\nabla u_{\lambda}\right|_{\mathrm{d}}(x)=\frac{p^{\prime}}{\alpha-1}\left(\lambda+\mathrm{d}\left(x_{0}, x\right)^{p^{\prime}}\right)^{\frac{\alpha}{1-\alpha}} \mathrm{d}\left(x_{0}, x\right)^{p^{\prime}-1}\left|\nabla \mathrm{d}\left(x_{0}, \cdot\right)\right|_{\mathrm{d}}(x), \quad x \in M .
$$

\section{Springer}


Since $\mathrm{d}\left(x_{0}, \cdot\right)$ is 1-Lipschitz (therefore, $\left|\nabla \mathrm{d}\left(x_{0}, \cdot\right)\right|_{\mathrm{d}}(x) \leq 1$ for all $x \in M$ ), due to (2.7), (2.8) and the form of the function $w_{G}$, we obtain the differential inequality

$$
\begin{aligned}
& \left(\frac{1-\alpha}{\alpha(p-1)+1} w_{G}^{\prime}(\lambda)\right)^{\frac{1}{\alpha p}} \\
& \quad \leq \mathcal{C}\left(\frac{p^{\prime}}{\alpha-1}\right)^{\theta}\left(w_{G}(\lambda)+\frac{\alpha-1}{\alpha(p-1)+1} \lambda w_{G}^{\prime}(\lambda)\right)^{\frac{\theta}{p}} w_{G}(\lambda)^{\frac{1-\theta}{\alpha(p-1)+1}} .
\end{aligned}
$$

Step 4 (Comparisonof $w_{G}$ andh $_{G}$ neartheorigin). We claim that

$$
\lim _{\lambda \rightarrow 0^{+}} \frac{w_{G}(\lambda)}{h_{G}(\lambda)}=1
$$

By hypothesis $(\mathbf{D})_{x_{0}}^{n}$, for every $\varepsilon>0$ there exists $\rho_{\varepsilon}>0$ such that

$$
\mathrm{m}\left(B_{x_{0}}(\rho)\right) \geq(1-\varepsilon) \omega_{n} \rho^{n} \text { for all } \rho \in\left[0, \rho_{\varepsilon}\right] .
$$

By (2.11), one has that

$$
\begin{aligned}
w_{G}(\lambda) & =\int_{0}^{\infty} \mathrm{m}\left(B_{x_{0}}(\rho)\right) f_{G}(\lambda, \rho) d \rho \\
& \geq(1-\varepsilon) \int_{0}^{\rho_{\varepsilon}} \omega_{n} \rho^{n} f_{G}(\lambda, \rho) d \rho=(1-\varepsilon) \lambda^{\frac{\alpha(p-1)+1}{1-\alpha}+\frac{n}{p^{\prime}}} \int_{0}^{\rho_{\varepsilon} \lambda^{-\frac{1}{p^{\prime}}}} \omega_{n} \rho^{n} f_{G}(1, \rho) d \rho .
\end{aligned}
$$

Thus, by the representation (2.3) of $h_{G}$ and a change of variables, it turns out that

$$
\liminf _{\lambda \rightarrow 0^{+}} \frac{w_{G}(\lambda)}{h_{G}(\lambda)} \geq(1-\varepsilon) \liminf _{\lambda \rightarrow 0^{+}} \frac{\int_{0}^{\rho_{\varepsilon} \lambda^{-\frac{1}{p^{\prime}}}} \omega_{n} \rho^{n} f_{G}(1, \rho) d \rho}{\int_{0}^{\infty} \omega_{n} \rho^{n} f_{G}(1, \rho) d \rho}=1-\varepsilon .
$$

The above inequality (with $\varepsilon>0$ arbitrary small) combined with (2.6) proves the claim (2.10).

Step 5 (Globalcomparisonof $w_{G}$ and $\left._{G}\right)$. We now claim that

$$
w_{G}(\lambda) \geq\left(\frac{\mathcal{G}_{\alpha, p, n}}{\mathcal{C}}\right)^{\frac{n}{\theta}} h_{G}(\lambda)=\tilde{h}_{G}(\lambda), \quad \lambda>0 .
$$

Since we assumed that $\mathcal{C}>\mathcal{G}_{\alpha, p, n}$, by (2.10) one has

$$
\lim _{\lambda \rightarrow 0^{+}} \frac{w_{G}(\lambda)}{\tilde{h}_{G}(\lambda)}=\left(\frac{\mathcal{C}}{\mathcal{G}_{\alpha, p, n}}\right)^{\frac{n}{\theta}}>1 .
$$

Therefore, there exists $\lambda_{0}>0$ such that for every $\lambda \in\left(0, \lambda_{0}\right)$, one has $w_{G}(\lambda)>\tilde{h}_{G}(\lambda)$.

By contradiction to (2.12), we assume that there exists $\lambda^{\#}>0$ such that $w_{G}\left(\lambda^{\#}\right)<\tilde{h}_{G}\left(\lambda^{\#}\right)$. If $\lambda^{*}=\sup \left\{0<\lambda<\lambda^{\#}: w_{G}(\lambda)=\tilde{h}_{G}(\lambda)\right\}$, then $0<\lambda_{0} \leq \lambda^{*}<\lambda^{\#}$. In particular,

$$
w_{G}(\lambda) \leq \tilde{h}_{G}(\lambda), \quad \forall \lambda \in\left[\lambda^{*}, \lambda^{\#}\right] .
$$


The latter relation and the differential inequality (2.9) imply that for every $\lambda \in\left[\lambda^{*}, \lambda^{\#}\right]$,

$$
\begin{aligned}
& \left(\frac{1-\alpha}{\alpha(p-1)+1} w_{G}^{\prime}(\lambda)\right)^{\frac{1}{\alpha \theta}} \\
& \leq \mathcal{C}^{\frac{p}{\theta}}\left(\frac{p^{\prime}}{\alpha-1}\right)^{p}\left(\tilde{h}_{G}(\lambda)+\frac{\alpha-1}{\alpha(p-1)+1} \lambda w_{G}^{\prime}(\lambda)\right) \tilde{h}_{G}(\lambda)^{\frac{(1-\theta) p}{\theta(\alpha(p-1)+1)}} .
\end{aligned}
$$

Moreover, since $\tilde{h}_{G}(\lambda)=\left(\frac{\mathcal{G}_{\alpha, p, b}}{\mathcal{C}}\right)^{\frac{n}{\theta}} h_{G}(\lambda)$, the ODE in (2.2) can be equivalently transformed for every $\lambda>0$ into the equation

$$
\begin{aligned}
& \left(\frac{1-\alpha}{\alpha(p-1)+1} \tilde{h}_{G}^{\prime}(\lambda)\right)^{\frac{1}{\alpha \theta}} \\
& =\mathcal{C}^{\frac{p}{\theta}}\left(\frac{p^{\prime}}{\alpha-1}\right)^{p}\left(\tilde{h}_{G}(\lambda)+\frac{\alpha-1}{\alpha(p-1)+1} \lambda \tilde{h}_{G}^{\prime}(\lambda)\right) \tilde{h}_{G}(\lambda)^{\frac{(1-\theta) p}{\theta(\alpha(p-1)+1)}}
\end{aligned}
$$

For $\lambda>0$ fixed we introduce the increasing function $j_{G}^{\lambda}:(0, \infty) \rightarrow \mathbb{R}$ defined by

$$
j_{G}^{\lambda}(t)=\left(\frac{\alpha-1}{\alpha(p-1)+1} t\right)^{\frac{1}{\alpha \theta}}+\mathcal{C}^{\frac{p}{\theta}}\left(\frac{p^{\prime}}{\alpha-1}\right)^{p} \frac{\alpha-1}{\alpha(p-1)+1} \lambda \tilde{h}_{G}(\lambda)^{\frac{(1-\theta) p}{\theta(\alpha(p-1)+1)}} t .
$$

Relations (2.13) and (2.14) can be rewritten into

$$
j_{G}^{\lambda}\left(-w_{G}^{\prime}(\lambda)\right) \leq \mathcal{C}^{\frac{p}{\theta}}\left(\frac{p^{\prime}}{\alpha-1}\right)^{p} \tilde{h}_{G}(\lambda)^{1+\frac{(1-\theta) p}{\theta(\alpha(p-1)+1)}}=j_{G}^{\lambda}\left(-\tilde{h}_{G}^{\prime}(\lambda)\right), \quad \forall \lambda \in\left[\lambda^{*}, \lambda^{\#}\right],
$$

which implies that

$$
-w_{G}^{\prime}(\lambda) \leq-\tilde{h}_{G}^{\prime}(\lambda), \quad \forall \lambda \in\left[\lambda^{*}, \lambda^{\#}\right]
$$

i.e., the function $\tilde{h}_{G}-w_{G}$ is non-increasing in $\left[\lambda^{*}, \lambda^{\#}\right]$. In particular, $0<\left(\tilde{h}_{G}-w_{G}\right)\left(\lambda^{\#}\right) \leq$ $\left(\tilde{h}_{G}-w_{G}\right)\left(\lambda^{*}\right)=0$, a contradiction. This concludes the proof of (2.12).

Step 6 (Asymptoticvolumegrowthestimatew.r.t. $x_{0}$ ). We claim that

$$
\ell_{\infty}^{x_{0}}:=\limsup _{\rho \rightarrow \infty} \frac{\mathrm{m}\left(B_{x_{0}}(\rho)\right)}{\omega_{n} \rho^{n}} \geq\left(\frac{\mathcal{G}_{\alpha, p, n}}{\mathcal{C}}\right)^{\frac{n}{\theta}} .
$$

By assuming the contrary, there exists $\varepsilon_{0}>0$ such that for some $\rho_{0}>0$,

$$
\frac{\mathrm{m}\left(B_{x_{0}}(\rho)\right)}{\omega_{n} \rho^{n}} \leq\left(\frac{\mathcal{G}_{\alpha, p, n}}{\mathcal{C}}\right)^{\frac{n}{\theta}}-\varepsilon_{0}, \quad \forall \rho \geq \rho_{0} .
$$

By (2.12) and from the latter relation, we have for every $\lambda>0$ that

$$
\begin{aligned}
0 & \leq w_{G}(\lambda)-\left(\frac{\mathcal{G}_{\alpha, p, n}}{\mathcal{C}}\right)^{\frac{n}{\theta}} h_{G}(\lambda) \\
& =\int_{0}^{\infty}\left(\frac{\mathrm{m}\left(B_{x_{0}}(\rho)\right)}{\omega_{n} \rho^{n}}-\left(\frac{\mathcal{G}_{\alpha, p, n}}{\mathcal{C}}\right)^{\frac{n}{\theta}}\right) \omega_{n} \rho^{n} f_{G}(\lambda, \rho) d \rho \\
& \leq\left(1+\varepsilon_{0}-\left(\frac{\mathcal{G}_{\alpha, p, n}}{\mathcal{C}}\right)^{\frac{n}{\theta}}\right) \int_{0}^{\rho_{0}} \omega_{n} \rho^{n} f_{G}(\lambda, \rho) d \rho-\varepsilon_{0} \int_{0}^{\infty} \omega_{n} \rho^{n} f_{G}(\lambda, \rho) d \rho
\end{aligned}
$$

Springer 
By using (2.3), a suitable rearrangement of the terms in the above relation shows that

$\varepsilon_{0} \frac{n}{p^{\prime}} \mathrm{B}\left(\frac{\alpha(p-1)+1}{\alpha-1}-\frac{n}{p^{\prime}}, \frac{n}{p^{\prime}}\right) \lambda^{1+\frac{n}{p^{\prime}}} \leq \frac{p^{\prime}}{n+p^{\prime}}\left(1+\varepsilon_{0}-\left(\frac{\mathcal{G}_{\alpha, p, n}}{\mathcal{C}}\right)^{\frac{n}{\theta}}\right) \frac{\alpha(p-1)+1}{\alpha-1} \rho_{0}^{n+p^{\prime}}$.

If we take the limit $\lambda \rightarrow+\infty$ in the last estimate, we obtain a contradiction. Thus, the claim (2.15) is proved and it remains to apply Lemma 2.1, which concludes the proof of Theorem 1.1 (i).

Proof of Theorem 1.1 (ii): the case $0<\alpha<1$. We shall invoke some of the arguments from the proof of Theorem 1.1 (i), emphasizing that subtle differences arise due to the 'dual' nature of the Gagliardo-Nirenberg inequalities $(\mathbf{G N 1})_{\mathcal{C}}^{\alpha, p}$ and $(\mathbf{G N 2})_{\mathcal{C}}^{\alpha, p}$, respectively. As before, we may assume that the inequality $(\mathbf{G N 2})_{\mathcal{C}}^{\alpha, p}$ holds with $\mathcal{C}>\mathcal{N}_{\alpha, p, n}$.

Step 1 The fact that $K=0$ works similarly as in Theorem 1.1 (i).

Step 2 Since $x \mapsto\left(\lambda^{p^{\prime}}-|x|^{p^{\prime}}\right)_{+}^{\frac{1}{1-\alpha}}$ is an extremal function in (1.3) for every $\lambda>0$, we obtain the ODE

$$
\begin{aligned}
h_{N}(\lambda)^{\frac{1}{\alpha(p-1)+1}}= & \mathcal{N}_{\alpha, p, n}\left(\frac{p^{\prime}}{1-\alpha}\right)^{\gamma}\left(-h_{N}(\lambda)+\frac{1-\alpha}{p^{\prime}(\alpha(p-1)+1)} \lambda h_{N}^{\prime}(\lambda)\right)^{\frac{\gamma}{p}} \times \\
& \times\left(\frac{1-\alpha}{p^{\prime}(\alpha(p-1)+1)} \lambda^{1-p^{\prime}} h_{N}^{\prime}(\lambda)\right)^{\frac{1-\gamma}{\alpha p}},
\end{aligned}
$$

where the function $h_{N}:(0, \infty) \rightarrow \mathbb{R}$ is defined by

$$
h_{N}(\lambda)=\int_{\mathbb{R}^{n}}\left(\lambda^{p^{\prime}}-|x|^{p^{\prime}}\right)_{+}^{\frac{\alpha(p-1)+1}{1-\alpha}} d x, \lambda>0 .
$$

It is clear that $h_{N}$ is well-defined, of class $C^{1}$ and can be represented as

$$
h_{N}(\lambda)=\omega_{n} \frac{n}{p^{\prime}} \mathrm{B}\left(\frac{\alpha(p-1)+1}{1-\alpha}+1, \frac{n}{p^{\prime}}\right) \lambda^{\frac{\alpha p p^{\prime}}{1-\alpha}+n+p^{\prime}}=\int_{0}^{\lambda} \omega_{n} \rho^{n} f_{N}(\lambda, \rho) d \rho,
$$

where

$$
f_{N}(\lambda, \rho)=p^{\prime} \frac{\alpha(p-1)+1}{1-\alpha}\left(\lambda^{p^{\prime}}-\rho^{p^{\prime}}\right)^{\frac{\alpha p}{1-\alpha}} \rho^{p^{\prime}-1}, \quad \text { for every } \lambda>0 \text { and } \rho \in(0, \lambda) .
$$

Step 3 Let $w_{N}:(0, \infty) \rightarrow \mathbb{R}$ be the function defined by

$$
w_{N}(\lambda)=\int_{M}\left(\lambda^{p^{\prime}}-\mathrm{d}\left(x_{0}, x\right)^{p^{\prime}}\right)_{+}^{\frac{\alpha(p-1)+1}{1-\alpha}} d \mathrm{~m}(x), \quad \lambda>0,
$$

where $x_{0} \in M$ is from (D) $)_{x_{0}}^{n}$. By the layer cake representation and relations (2.5) and (2.17), $w_{N}$ is well-defined, positive, of class $C^{1}$ and

$$
0<w_{N}(\lambda)=\int_{0}^{\lambda} \mathrm{m}\left(B_{x_{0}}(\rho)\right) f_{N}(\lambda, \rho) d \rho \leq \int_{0}^{\lambda} \omega_{n} \rho^{n} f_{N}(\lambda, \rho) d \rho=h_{N}(\lambda)<\infty, \lambda>0 .
$$

Since $u_{\lambda}=\left(\lambda^{p^{\prime}}-\mathrm{d}\left(x_{0}, \cdot\right)^{p^{\prime}}\right)_{+}^{\frac{1}{1-\alpha}}$ is a Lipschitz function on $M$ with compact support $\overline{B_{x_{0}}(\lambda)}$, it belongs to $\operatorname{Lip}_{0}(M)$. Therefore, we may apply $u_{\lambda}$ in $(\mathbf{G N 2})_{\mathcal{C}}^{\alpha, p}$; a similar reasoning as in (2.8) leads to the differential inequality 


$$
\begin{aligned}
w_{N}(\lambda)^{\frac{1}{\alpha(p-1)+1} \leq} & \mathcal{C}\left(\frac{p^{\prime}}{1-\alpha}\right)^{\gamma}\left(-w_{N}(\lambda)+\frac{1-\alpha}{p^{\prime}(\alpha(p-1)+1)} \lambda w_{N}^{\prime}(\lambda)\right)^{\frac{\gamma}{p}} \\
& \times\left(\frac{1-\alpha}{p^{\prime}(\alpha(p-1)+1)} \lambda^{1-p^{\prime}} w_{N}^{\prime}(\lambda)\right)^{\frac{1-\gamma}{\alpha p}}, \quad \lambda>0 .
\end{aligned}
$$

Step 4 For an arbitrarily fixed $\varepsilon>0$, let $\rho_{\varepsilon}>0$ from (2.11). If $0<\lambda<\rho_{\varepsilon}$, one has that $w_{N}(\lambda)=\int_{0}^{\lambda} \mathrm{m}\left(B_{x_{0}}(\rho)\right) f_{N}(\lambda, \rho) d \rho \geq(1-\varepsilon) \int_{0}^{\lambda} \omega_{n} \rho^{n} f_{N}(\lambda, \rho) d \rho=(1-\varepsilon) h_{N}(\lambda)$.

Consequently, the latter relation together with (2.18) implies that

$$
\lim _{\lambda \rightarrow 0^{+}} \frac{w_{N}(\lambda)}{h_{N}(\lambda)}=1
$$

Step 5 We shall prove that

$$
w_{N}(\lambda) \geq\left(\frac{\mathcal{N}_{\alpha, p, n}}{\mathcal{C}}\right)^{\frac{n}{\gamma}} h_{N}(\lambda)=\tilde{h}_{N}(\lambda), \quad \lambda>0 .
$$

By (2.20) one has

$$
\lim _{\lambda \rightarrow 0^{+}} \frac{w_{N}(\lambda)}{\tilde{h}_{N}(\lambda)}=\left(\frac{\mathcal{C}}{\mathcal{N}_{\alpha, p, n}}\right)^{\frac{n}{\gamma}}>1
$$

which implies the existence of a number $\lambda_{0}>0$ such that $w_{N}(\lambda)>\tilde{h}_{N}(\lambda)$ for every $\lambda \in\left(0, \lambda_{0}\right)$.

We assume by contradiction that there exists $\lambda^{\#}>0$ such that $w_{N}\left(\lambda^{\#}\right)<\tilde{h}_{N}\left(\lambda^{\#}\right)$. If $\lambda^{*}=\sup \left\{0<\lambda<\lambda^{\#}: w_{N}(\lambda)=\tilde{h}_{N}(\lambda)\right\}$, then $0<\lambda_{0} \leq \lambda^{*}<\lambda^{\#}$ and

$$
w_{N}(\lambda) \leq \tilde{h}_{N}(\lambda), \quad \forall \lambda \in\left[\lambda^{*}, \lambda^{\#}\right] .
$$

For every $\lambda>0$, let $j_{N}^{\lambda}:\left(\frac{p^{\prime}(\alpha(p-1)+1)}{(1-\alpha) \lambda}, \infty\right) \rightarrow \mathbb{R}$ be the function defined by

$$
j_{N}^{\lambda}(t)=\mathcal{C}\left(\frac{p^{\prime}}{1-\alpha}\right)^{\gamma}\left(-1+\frac{1-\alpha}{p^{\prime}(\alpha(p-1)+1)} \lambda t\right)^{\frac{\gamma}{p}}\left(\frac{1-\alpha}{p^{\prime}(\alpha(p-1)+1)} \lambda^{1-p^{\prime}} t\right)^{\frac{1-\gamma}{\alpha p}} .
$$

It is clear that $j_{N}^{\lambda}$ is well-defined, positive and increasing. A direct computation yields that both values $\left(\log w_{N}\right)^{\prime}(\lambda)=\frac{w_{N}^{\prime}(\lambda)}{w_{N}(\lambda)}$ and $\left(\log \tilde{h}_{N}\right)^{\prime}(\lambda)=\frac{\tilde{h}_{N}^{\prime}(\lambda)}{\tilde{h}_{N}(\lambda)}$ are greater than $\frac{p^{\prime}(\alpha(p-1)+1)}{(1-\alpha) \lambda}$ for every $\lambda>0$. Taking into account (1.4), we have

$$
\frac{1}{\alpha(p-1)+1}-\frac{\gamma}{p}-\frac{1-\gamma}{\alpha p}=-\frac{\gamma}{n}
$$

therefore, if we divide the inequality (2.19) by $w_{N}(\lambda)^{\frac{\gamma}{p}+\frac{1-\gamma}{\alpha p}}$, we obtain that

$$
w_{N}(\lambda)^{-\frac{\gamma}{n}} \leq j_{N}^{\lambda}\left(\left(\log w_{N}\right)^{\prime}(\lambda)\right), \quad \forall \lambda>0 .
$$

In a similar manner, by $\tilde{h}_{N}(\lambda)=\left(\frac{\mathcal{N}_{\alpha, p, n}}{\mathcal{C}}\right)^{\frac{n}{\gamma}} h_{N}(\lambda)$ and relation $(2.16)$, we have that

$$
\tilde{h}_{N}(\lambda)^{-\frac{\gamma}{n}}=j_{N}^{\lambda}\left(\left(\log \tilde{h}_{N}\right)^{\prime}(\lambda)\right), \quad \forall \lambda>0 .
$$

\section{Springer}


Thus, by (2.22), (2.23) and (2.24), it turns out that

$$
j_{N}^{\lambda}\left(\left(\log \tilde{h}_{N}\right)^{\prime}(\lambda)\right)=\tilde{h}_{N}(\lambda)^{-\frac{\gamma}{n}} \leq w_{N}(\lambda)^{-\frac{\gamma}{n}} \leq j_{N}^{\lambda}\left(\left(\log w_{N}\right)^{\prime}(\lambda)\right), \quad \forall \lambda \in\left[\lambda^{*}, \lambda^{\#}\right] .
$$

Since the inverse of $j_{N}^{\lambda}$ is also increasing, it follows that $\left(\log \tilde{h}_{N}\right)^{\prime}(\lambda) \leq\left(\log w_{N}\right)^{\prime}(\lambda)$ for every $\lambda \in\left[\lambda^{*}, \lambda^{\#}\right]$. Therefore, the function $\lambda \mapsto \log \frac{\tilde{h}_{N}(\lambda)}{w_{N}(\lambda)}$ is non-increasing in the interval $\left[\lambda^{*}, \lambda^{\#}\right]$. In particular, it follows that

$$
0<\log \frac{\tilde{h}_{N}\left(\lambda^{\#}\right)}{w_{N}\left(\lambda^{\#}\right)} \leq \log \frac{\tilde{h}_{N}\left(\lambda^{*}\right)}{w_{N}\left(\lambda^{*}\right)}=0,
$$

a contradiction, which proves the validity of the claim (2.21).

Step 6 We shall prove that

$$
\limsup _{\rho \rightarrow \infty} \frac{\mathrm{m}\left(B_{x_{0}}(\rho)\right)}{\omega_{n} \rho^{n}} \geq\left(\frac{\mathcal{N}_{\alpha, p, n}}{\mathcal{C}}\right)^{\frac{n}{\gamma}}
$$

By contradiction, we assume that there exists $\varepsilon_{0}>0$ such that for some $\rho_{0}>0$,

$$
\frac{\mathrm{m}\left(B_{x_{0}}(\rho)\right)}{\omega_{n} \rho^{n}} \leq\left(\frac{\mathcal{N}_{\alpha, p, n}}{\mathcal{C}}\right)^{\frac{n}{\gamma}}-\varepsilon_{0}, \quad \forall \rho \geq \rho_{0} .
$$

The above inequality and (2.21) imply that for every $\lambda>\rho_{0}$,

$$
\begin{aligned}
0 & \leq w_{N}(\lambda)-\left(\frac{\mathcal{N}_{\alpha, p, n}}{\mathcal{C}}\right)^{\frac{n}{\gamma}} h_{N}(\lambda)=\int_{0}^{\lambda}\left(\frac{\mathrm{m}\left(B_{x_{0}}(\rho)\right)}{\omega_{n} \rho^{n}}-\left(\frac{\mathcal{N}_{\alpha, p, n}}{\mathcal{C}}\right)^{\frac{n}{\gamma}}\right) \omega_{n} \rho^{n} f_{N}(\lambda, \rho) d \rho \\
& \leq\left(1+\varepsilon_{0}-\left(\frac{\mathcal{N}_{\alpha, p, n}}{\mathcal{C}}\right)^{\frac{n}{\gamma}}\right) \int_{0}^{\rho_{0}} \omega_{n} \rho^{n} f_{N}(\lambda, \rho) d \rho-\varepsilon_{0} \int_{0}^{\lambda} \omega_{n} \rho^{n} f_{N}(\lambda, \rho) d \rho .
\end{aligned}
$$

Reorganizing the latter estimate, it follows that for every $\lambda>0$,

$$
\begin{aligned}
\varepsilon_{0} & \frac{n}{p^{\prime}} \mathrm{B}\left(\frac{\alpha(p-1)+1}{1-\alpha}+1, \frac{n}{p^{\prime}}\right) \lambda^{n+p^{\prime}} \\
\leq & \frac{p^{\prime}}{n+p^{\prime}}\left(1+\varepsilon_{0}-\left(\frac{\mathcal{N}_{\alpha, p, n}}{\mathcal{C}}\right)^{\frac{n}{\gamma}}\right) \frac{\alpha(p-1)+1}{1-\alpha} \rho_{0}^{n+p^{\prime}} .
\end{aligned}
$$

Once we let $\lambda \rightarrow \infty$, we get a contradiction. Therefore, (2.25) holds and Lemma 2.1 yields that

$$
\frac{\mathrm{m}\left(B_{x}(\rho)\right)}{\omega_{n} \rho^{n}} \geq\left(\frac{\mathcal{N}_{\alpha, p, n}}{\mathcal{C}}\right)^{\frac{n}{\gamma}}, \quad \forall x \in M, \rho>0,
$$

which concludes the proof of Theorem 1.1 (ii).

\subsection{Limit case I $(\alpha \rightarrow 1): L^{p}$-logarithmic Sobolev inequality}

In this subsection we shall provide the proof of Theorem 1.2. We shall assume that $\mathcal{C}>\mathcal{L}_{p, n}$ in $(\mathbf{L S})_{\mathcal{C}}^{p}$.

Step 1 As in the previous proofs, we obtain that $K=0$; the only difference is that we shall consider $u(x)=\mathrm{m}(M)^{-1 / p}$ as a test function in $(\mathbf{L S})_{\mathcal{C}}^{p}$, in order to fulfil the normalization assumption $\|u\|_{L^{p}}=1$. 
Step 2 Since the functions $l_{p}^{\lambda}(\lambda>0)$ in Theorem B are extremals in (1.5), once we plug them we obtain a first order ODE of the form

$$
-\log h_{L}(\lambda)+\lambda \frac{h_{L}^{\prime}(\lambda)}{h_{L}(\lambda)}=\frac{n}{p} \log \left(-\mathcal{L}_{p, n}\left(\frac{p^{\prime}}{p}\right)^{p} \lambda^{p} \frac{h_{L}^{\prime}(\lambda)}{h_{L}(\lambda)}\right), \quad \lambda>0,
$$

where $h_{L}:(0, \infty) \rightarrow \mathbb{R}$ is defined by

$$
h_{L}(\lambda)=\int_{\mathbb{R}^{n}} e^{-\lambda|x|^{p^{\prime}}} d x
$$

For later use, we recall that $h_{L}$ can be represented alternatively by

$h_{L}(\lambda)=\frac{2 \pi^{\frac{n}{2}}}{p^{\prime} \lambda^{\frac{n}{p^{\prime}}}} \cdot \frac{\Gamma\left(\frac{n}{p^{\prime}}\right)}{\Gamma\left(\frac{n}{2}\right)}=\lambda p^{\prime} \omega_{n} \int_{0}^{\infty} e^{-\lambda \rho^{p^{\prime}}} \rho^{n+p^{\prime}-1} d \rho=\lambda^{-\frac{n}{p^{\prime}}} p^{\prime} \omega_{n} \int_{0}^{\infty} e^{-t^{p^{\prime}}} t^{n+p^{\prime}-1} d t$.

Step 3 Let $w_{L}:(0, \infty) \rightarrow \mathbb{R}$ be defined by

$$
w_{L}(\lambda)=\int_{M} e^{-\lambda \mathrm{d}\left(x_{0}, x\right)^{p^{\prime}}} d \mathrm{~m}(x)
$$

where $x_{0} \in M$ is the element from hypothesis $(\mathbf{D})_{x_{0}}^{n}$. Note that $w_{L}$ is well-defined, positive and differentiable. Indeed, by the layer cake representation, for every $\lambda>0$ we obtain that

$$
\begin{array}{rlrl}
w_{L}(\lambda) & =\int_{0}^{\infty} \mathrm{m}\left(\left\{x \in M: e^{-\lambda \mathrm{d}\left(x_{0}, x\right)^{p^{\prime}}}>t\right\}\right) d t=\int_{0}^{1} \mathrm{~m}\left(\left\{x \in M: e^{-\lambda \mathrm{d}\left(x_{0}, x\right)^{p^{\prime}}}>t\right\}\right) d t \\
& =\lambda p^{\prime} \int_{0}^{\infty} \mathrm{m}\left(B_{x_{0}}(\rho)\right) e^{-\lambda \rho^{p^{\prime}}} \rho^{p^{\prime}-1} d \rho & \text { [change } \left.t=e^{-\lambda \rho^{p^{\prime}}}\right] \\
& \leq \lambda p^{\prime} \omega_{n} \int_{0}^{\infty} e^{-\lambda \rho^{p^{\prime}}} \rho^{n+p^{\prime}-1} d \rho & \text { [see (2.5)] } \\
& =h_{L}(\lambda)<+\infty . &
\end{array}
$$

Let us consider the family of functions $\tilde{u}_{\lambda}: M \rightarrow \mathbb{R}(\lambda>0)$ defined by

$$
\tilde{u}_{\lambda}(x)=\frac{e^{-\frac{\lambda}{p} \mathrm{~d}\left(x_{0}, x\right)^{p^{\prime}}}}{w_{L}(\lambda)^{\frac{1}{p}}}, \quad x \in M .
$$

It is clear that $\left\|\tilde{u}_{\lambda}\right\|_{L^{p}}=1$ and as in the proof of Theorem 1.1 (i), the function $\tilde{u}_{\lambda}$ can be approximated by elements from $\operatorname{Lip}_{0}(M)$; in fact, $\tilde{u}_{\lambda}$ can be used as a test function in $(\mathbf{L S})_{\mathcal{C}}^{p}$. Thus, plugging $\tilde{u}_{\lambda}$ into the inequality $(\mathbf{L S})_{\mathcal{C}}^{p}$, applying the non-smooth chain rule and the fact that $\left|\nabla \mathrm{d}\left(x_{0}, \cdot\right)\right|_{\mathrm{d}}(x) \leq 1$ for every $x \in M$, it yields

$$
-\log w_{L}(\lambda)+\lambda \frac{w_{L}^{\prime}(\lambda)}{w_{L}(\lambda)} \leq \frac{n}{p} \log \left(-\mathcal{C}\left(\frac{p^{\prime}}{p}\right)^{p} \lambda^{p} \frac{w_{L}^{\prime}(\lambda)}{w_{L}(\lambda)}\right), \quad \lambda>0 .
$$

Step 4 We prove that

$$
\lim _{\lambda \rightarrow+\infty} \frac{w_{L}(\lambda)}{h_{L}(\lambda)}=1
$$

\section{를 Springer}


For a fixed $\varepsilon>0$, let $\rho_{\varepsilon}>0$ from (2.11). Then one has

$$
\begin{array}{rlrl}
w_{L}(\lambda) & =\lambda p^{\prime} \int_{0}^{\infty} \mathrm{m}\left(B_{x_{0}}(\rho)\right) e^{-\lambda \rho^{p^{\prime}}} \rho^{p^{\prime}-1} d \rho \geq \lambda p^{\prime}(1-\varepsilon) \omega_{n} \int_{0}^{\rho_{\varepsilon}} e^{-\lambda \rho^{p^{\prime}}} \rho^{n+p^{\prime}-1} d \rho \\
& =\lambda^{-\frac{n}{p^{\prime}}} p^{\prime}(1-\varepsilon) \omega_{n} \int_{0}^{\rho_{\varepsilon} \lambda^{\frac{1}{p^{\prime}}}} e^{-t^{p^{\prime}}} t^{n+p^{\prime}-1} d t . & \text { [change } t=\lambda^{\frac{1}{p^{\prime}}} \rho \text { ] }
\end{array}
$$

Therefore, by the third representation of $h_{L}$ (see (2.27)) it turns out that

$$
\liminf _{\lambda \rightarrow+\infty} \frac{w_{L}(\lambda)}{h_{L}(\lambda)} \geq 1-\varepsilon .
$$

The arbitrariness of $\varepsilon>0$ together with Step 3 implies the validity of (2.29).

Step 5 We claim that

$$
w_{L}(\lambda) \geq\left(\frac{\mathcal{L}_{p, n}}{\mathcal{C}}\right)^{\frac{n}{p}} h_{L}(\lambda)=: \tilde{h}_{L}(\lambda), \quad \lambda>0 .
$$

Since $\mathcal{C}>\mathcal{L}_{p, n}$, by (2.29) it follows that

$$
\lim _{\lambda \rightarrow+\infty} \frac{w_{L}(\lambda)}{\tilde{h}_{L}(\lambda)}=\left(\frac{\mathcal{C}}{\mathcal{L}_{p, n}}\right)^{\frac{n}{p}}>1 .
$$

Consequently, there exists $\tilde{\lambda}>0$ such that $w_{L}(\lambda)>\tilde{h}_{L}(\lambda)$ for all $\lambda>\tilde{\lambda}$. If we introduce the notations

$$
W(\lambda)=\log w_{L}(\lambda) \text { and } \tilde{H}(\lambda)=\log \tilde{h}_{L}(\lambda), \quad \lambda>0,
$$

the latter relation implies that

$$
W(\lambda)>\tilde{H}(\lambda), \quad \forall \lambda>\tilde{\lambda},
$$

while relations in (2.28) and (2.26) can be rewritten in terms of $W$ and $\tilde{H}$ as

$$
-W(\lambda)+\lambda W^{\prime}(\lambda) \leq \frac{n}{p} \log \left(-\mathcal{C}\left(\frac{p^{\prime}}{p}\right)^{p} \lambda^{p} W^{\prime}(\lambda)\right), \quad \lambda>0,
$$

and

$$
-\tilde{H}(\lambda)+\lambda \tilde{H}^{\prime}(\lambda)=\frac{n}{p} \log \left(-\mathcal{C}\left(\frac{p^{\prime}}{p}\right)^{p} \lambda^{p} \tilde{H}^{\prime}(\lambda)\right), \quad \lambda>0 .
$$

Claim (2.30) is proved once we show that $W(\lambda) \geq \tilde{H}(\lambda)$ for all $\lambda>0$. By contradiction, we assume there exists $\lambda^{\#}>0$ such that $W\left(\lambda^{\#}\right)<\tilde{H}\left(\lambda^{\#}\right)$. Due to $(2.31), \lambda^{\#}<\tilde{\lambda}$. On the one hand, let $\lambda^{*}=\inf \left\{\lambda>\lambda^{\#}: W(\lambda)=\tilde{H}(\lambda)\right\}$. In particular,

$$
W(\lambda) \leq \tilde{H}(\lambda), \quad \forall \lambda \in\left[\lambda^{\#}, \lambda^{*}\right] .
$$

On the other hand, if we introduce for every $\lambda>0$ the function $j_{L}^{\lambda}:(0, \infty) \rightarrow \mathbb{R}$ by

$$
j_{L}^{\lambda}(t)=\frac{n}{p} \log \left(\mathcal{C}\left(\frac{p^{\prime}}{p}\right)^{p} \lambda^{p} t\right)+\lambda t, \quad t>0,
$$

relations (2.32) and (2.33) become

$$
-W(\lambda) \leq j_{L}^{\lambda}\left(-W^{\prime}(\lambda)\right) \text { and }-\tilde{H}(\lambda)=j_{L}^{\lambda}\left(-\tilde{H}^{\prime}(\lambda)\right), \quad \lambda>0 .
$$


By the above relations and (2.34) it yields that

$$
j_{L}^{\lambda}\left(-\tilde{H}^{\prime}(\lambda)\right)=-\tilde{H}(\lambda) \leq-W(\lambda) \leq j_{L}^{\lambda}\left(-W^{\prime}(\lambda)\right), \quad \forall \lambda \in\left[\lambda^{\#}, \lambda^{*}\right] .
$$

Since $j_{L}^{\lambda}$ is increasing, it follows that $W-\tilde{H}$ is a non-increasing function on $\left[\lambda^{\#}, \lambda^{*}\right]$, which implies

$$
0=(W-\tilde{H})\left(\lambda^{*}\right) \leq(W-\tilde{H})\left(\lambda^{\#}\right)<0,
$$

a contradiction. This completes the proof of (2.30).

Step 6 We claim that

$$
\limsup _{\rho \rightarrow \infty} \frac{\mathrm{m}\left(B_{x_{0}}(\rho)\right)}{\omega_{n} \rho^{n}} \geq\left(\frac{\mathcal{L}_{p, n}}{\mathcal{C}}\right)^{\frac{n}{p}} .
$$

By assuming the contrary, there exists $\varepsilon_{0}>0$ such that for some $\rho_{0}>0$,

$$
\frac{\mathrm{m}\left(B_{x_{0}}(\rho)\right)}{\omega_{n} \rho^{n}} \leq\left(\frac{\mathcal{L}_{p, n}}{\mathcal{C}}\right)^{\frac{n}{p}}-\varepsilon_{0}, \quad \forall \rho \geq \rho_{0} .
$$

Combining the latter relation with (2.30) and (2.27), for every $\lambda>0$ we obtain that

$0 \leq w_{L}(\lambda)-\left(\frac{\mathcal{L}_{p, n}}{\mathcal{C}}\right)^{\frac{n}{p}} h_{L}(\lambda)$

$$
\leq \lambda p^{\prime} \int_{0}^{\rho_{0}} \mathrm{~m}\left(B_{x_{0}}(\rho)\right) e^{-\lambda \rho^{p^{\prime}}} \rho^{p^{\prime}-1} d \rho+\lambda p^{\prime} \omega_{n}\left(\left(\frac{\mathcal{L}_{p, n}}{\mathcal{C}}\right)^{\frac{n}{p}}-\varepsilon_{0}\right) \int_{\rho_{0}}^{\infty} e^{-\lambda \rho^{p^{\prime}}} \rho^{n+p^{\prime}-1} d \rho
$$$$
-\lambda p^{\prime} \omega_{n}\left(\frac{\mathcal{L}_{p, n}}{\mathcal{C}}\right)^{\frac{n}{p}} \int_{0}^{\infty} e^{-\lambda \rho^{p^{\prime}}} \rho^{n+p^{\prime}-1} d \rho .
$$

Rearranging the above inequality, by virtue of (2.5) it follows for every $\lambda>0$ that

$$
\varepsilon_{0} \int_{0}^{\infty} e^{-\lambda \rho^{p^{\prime}}} \rho^{n+p^{\prime}-1} d \rho \leq\left(1-\left(\frac{\mathcal{L}_{p, n}}{\mathcal{C}}\right)^{\frac{n}{p}}+\varepsilon_{0}\right) \int_{0}^{\rho_{0}} e^{-\lambda \rho^{p^{\prime}}} \rho^{n+p^{\prime}-1} d \rho .
$$

Due to (2.27), the latter inequality implies

$$
\varepsilon_{0} \frac{1}{p^{\prime} \lambda^{1+\frac{n}{p^{\prime}}}} \Gamma\left(\frac{n}{p^{\prime}}+1\right) \leq\left(1-\left(\frac{\mathcal{L}_{p, n}}{\mathcal{C}}\right)^{\frac{n}{p}}+\varepsilon_{0}\right) \frac{\rho_{0}^{n+p^{\prime}}}{n+p^{\prime}}, \lambda>0 .
$$

Now, letting $\lambda \rightarrow 0^{+}$we arrive to a contradiction. Therefore, the proof of (2.35) is concluded. Thus, Lemma 2.1 gives that

$$
\frac{\mathrm{m}\left(B_{x}(\rho)\right)}{\omega_{n} \rho^{n}} \geq\left(\frac{\mathcal{L}_{p, n}}{\mathcal{C}}\right)^{\frac{n}{p}}, \quad \forall x \in M, \quad \rho>0,
$$

concluding the proof of Theorem 1.2.

\subsection{Limit case II $(\alpha \rightarrow 0)$ : Faber-Krahn-type inequality}

In this part we sketch the proof of Theorem 1.3. Similarly as before, we assume that $\mathcal{C}>\mathcal{F}_{p, n}$. Step 1 Analogously to Theorem 1.1 (i), it follows that $K=0$.

\section{国 Springer}


Step 2 The function $x \mapsto\left(\lambda^{p^{\prime}}-|x|^{p^{\prime}}\right)_{+}$being extremal in (1.6) for every $\lambda>0$, a direct computation shows that

$$
h_{F}(\lambda)=\mathcal{F}_{p, n} p^{\prime}\left(-h_{F}(\lambda)+\frac{1}{p^{\prime}} \lambda h_{F}^{\prime}(\lambda)\right)^{\frac{1}{p}}\left(\frac{1}{p^{\prime}} \lambda^{1-p^{\prime}} h_{F}^{\prime}(\lambda)\right)^{1-\frac{1}{p^{\star}}}
$$

where $h_{F}:(0, \infty) \rightarrow \mathbb{R}$ is given by

$$
h_{F}(\lambda)=\int_{\mathbb{R}^{n}}\left(\lambda^{p^{\prime}}-|x|^{p^{\prime}}\right)_{+} d x, \lambda>0
$$

Step 3 Let $x_{0} \in M$ from $(\mathbf{D})_{x_{0}}^{n}$. Since $u_{\lambda}=\left(\lambda^{p^{\prime}}-\mathrm{d}\left(x_{0}, \cdot\right)^{p^{\prime}}\right)_{+} \in \operatorname{Lip}_{0}(M)$, we may insert $u_{\lambda}$ into $(\mathbf{F K})_{\mathcal{C}}^{p}$ obtaining

$$
\left\|u_{\lambda}\right\|_{L^{1}} \leq \mathcal{C}\left\|\left|\nabla u_{\lambda}\right|_{\mathrm{d}}\right\|_{L^{p}} \mathrm{~m}\left(\operatorname{supp}\left(u_{\lambda}\right)\right)^{1-\frac{1}{p^{\star}}} .
$$

First, we observe that

$$
\left|\nabla u_{\lambda}\right|_{\mathrm{d}}(x)=p^{\prime} \mathrm{d}\left(x_{0}, x\right)^{p^{\prime}-1}\left|\nabla \mathrm{d}\left(x_{0}, \cdot\right)\right|_{\mathrm{d}}(x) \leq p^{\prime} \mathrm{d}\left(x_{0}, x\right)^{p^{\prime}-1}, \quad \forall x \in B_{x_{0}}(\lambda),
$$

while $\left|\nabla u_{\lambda}\right|_{d}(x)=0$ for every $x \notin B_{x_{0}}(\lambda)$. Moreover, since the spheres have zero m-measures (see Theorem 2.1), we have that

$$
\mathrm{m}\left(\operatorname{supp}\left(u_{\lambda}\right)\right)=\mathrm{m}\left(\overline{B_{x_{0}}(\lambda)}\right)=\mathrm{m}\left(B_{x_{0}}(\lambda)\right) .
$$

We now introduce the function $w_{F}:(0, \infty) \rightarrow \mathbb{R}$ given by

$$
w_{F}(\lambda)=\int_{M}\left(\lambda^{p^{\prime}}-\mathrm{d}\left(x_{0}, x\right)^{p^{\prime}}\right)_{+} d \mathrm{~m}(x), \lambda>0 .
$$

Due to the layer cake representation, one has

$$
\begin{aligned}
w_{F}(\lambda) & =\int_{B_{x_{0}}(\lambda)}\left(\lambda^{p^{\prime}}-\mathrm{d}\left(x_{0}, x\right)^{p^{\prime}}\right) d \mathrm{~m}(x)=\lambda^{p^{\prime}} \mathrm{m}\left(B_{x_{0}}(\lambda)\right)-\int_{B_{x_{0}}(\lambda)} \mathrm{d}\left(x_{0}, x\right)^{p^{\prime}} d \mathrm{~m}(x) \\
& =\lambda^{p^{\prime}} \mathrm{m}\left(B_{x_{0}}(\lambda)\right)-\int_{0}^{\lambda^{p^{\prime}}} \mathrm{m}\left(\left\{x \in B_{x_{0}}(\lambda): \mathrm{d}\left(x_{0}, x\right)^{p^{\prime}}>t\right\}\right) d t \\
& \left.=\lambda^{p^{\prime}} \mathrm{m}\left(B_{x_{0}}(\lambda)\right)-p^{\prime} \int_{0}^{\lambda}\left(\mathrm{m}\left(B_{x_{0}}(\lambda)\right)-\mathrm{m}\left(B_{x_{0}}(\rho)\right)\right) \rho^{p^{\prime}-1} d \rho \quad \text { [change } t=\rho^{p^{\prime}}\right] \\
& =p^{\prime} \int_{0}^{\lambda} \mathrm{m}\left(B_{x_{0}}(\rho)\right) \rho^{p^{\prime}-1} d \rho .
\end{aligned}
$$

Therefore,

$$
\left\|u_{\lambda}\right\|_{L^{1}}=w_{F}(\lambda), \mathrm{m}\left(\operatorname{supp}\left(u_{\lambda}\right)\right)=\mathrm{m}\left(B_{x_{0}}(\lambda)\right)=\frac{1}{p^{\prime}} \lambda^{1-p^{\prime}} w_{F}^{\prime}(\lambda),
$$

and

$$
\left\|\left|\nabla u_{\lambda}\right|_{\mathrm{d}}\right\|_{L^{p}} \leq p^{\prime}\left(\int_{B_{x_{0}}(\lambda)} \mathrm{d}\left(x_{0}, x\right)^{p^{\prime}} d \mathrm{~m}(x)\right)^{\frac{1}{p}}=p^{\prime}\left(-w_{F}(\lambda)+\frac{1}{p^{\prime}} \lambda w_{F}^{\prime}(\lambda)\right)^{\frac{1}{p}} .
$$

Consequently, inequality (2.37) takes the form

$$
w_{F}(\lambda) \leq \mathcal{C} p^{\prime}\left(-w_{F}(\lambda)+\frac{1}{p^{\prime}} \lambda w_{F}^{\prime}(\lambda)\right)^{\frac{1}{p}}\left(\frac{1}{p^{\prime}} \lambda^{1-p^{\prime}} w_{F}^{\prime}(\lambda)\right)^{1-\frac{1}{p^{\star}}}, \lambda>0,
$$


which is formally (2.19) if $\alpha \rightarrow 0$ since due to (1.4), $\lim _{\alpha \rightarrow 0} \gamma=1$ and $\lim _{\alpha \rightarrow 0} \frac{1-\gamma}{\alpha p}=$ $1-\frac{1}{p^{\star}}$.

Therefore, we may proceed as in the proof of Theorem 1.1 (ii) (Steps 4-6), proving that

$$
\lim _{\lambda \rightarrow 0^{+}} \frac{w_{F}(\lambda)}{h_{F}(\lambda)}=1
$$

$$
w_{F}(\lambda) \geq\left(\frac{\mathcal{F}_{p, n}}{\mathcal{C}}\right)^{n} h_{F}(\lambda), \quad \forall \lambda>0,
$$

and finally

$$
\frac{\mathrm{m}\left(B_{x}(\rho)\right)}{\omega_{n} \rho^{n}} \geq\left(\frac{\mathcal{F}_{p, n}}{\mathcal{C}}\right)^{n}, \quad \forall x \in M, \rho>0,
$$

which concludes the proof of Theorem 1.3.

\section{Rigidity results in smooth settings}

As a starting point, we need an Aubin-Hebey-type result (see [3] and [11]) for GagliardoNirenberg inequalities which is valid on generic Riemannian manifolds.

Lemma 3.1 Let $(M, g)$ be a complete $n$-dimensional Riemannian manifold and $\mathcal{C}>0$. The following statements hold:

(i) If (GN1) $)_{\mathcal{C}}^{\alpha, p}$ holds on $(M, g)$ for some $p \in(1, n)$ and $\alpha \in\left(1, \frac{n}{n-p}\right]$ then $\mathcal{C} \geq \mathcal{G}_{\alpha, p, n}$;

(ii) If (GN2 $)_{\mathcal{C}}^{\alpha, p}$ holds on $(M, g)$ for some $p \in(1, n)$ and $\alpha \in(0,1)$ then $\mathcal{C} \geq \mathcal{N}_{\alpha, p, n}$;

(iii) If $(\mathbf{L S})_{\mathcal{C}}^{p}$ holds on $(M, g)$ for some $p \in(1, n)$ then $\mathcal{C} \geq \mathcal{L}_{p, n}$;

(iv) If (FK) $)_{\mathcal{C}}^{p}$ holds on $(M, g)$ for some $p \in(1, n)$ then $\mathcal{C} \geq \mathcal{F}_{p, n}$.

Proof (i) By contradiction, we assume that $(\mathbf{G N 1})_{\mathcal{C}}^{\alpha, p}$ holds on $(M, g)$ for some $p \in(1, n)$, $\alpha \in\left(1, \frac{n}{n-p}\right]$, and $\mathcal{C}<\mathcal{G}_{\alpha, p, n}$. Let $x_{0} \in M$ be fixed arbitrarily. For every $\varepsilon>0$, there exists a local chart $(\Omega, \phi)$ of $M$ at the point $x_{0}$ and a number $\delta>0$ such that $\phi(\Omega)=B_{0}(\delta)=$ $\left\{\tilde{x} \in \mathbb{R}^{n}:|\tilde{x}|<\delta\right\}$ and the components $g_{i j}=g_{i j}(x)$ of the Riemannian metric $g$ on $(\Omega, \phi)$ satisfy

$$
(1-\varepsilon) \delta_{i j} \leq g_{i j} \leq(1+\varepsilon) \delta_{i j}
$$

in the sense of bilinear forms. Since $(\mathbf{G N 1})_{\mathcal{C}}^{\alpha, p}$ is valid, relation (3.1) shows that for every $\varepsilon>0$ small enough, there exists $\delta_{\varepsilon}>0$ and $\mathcal{C}_{\varepsilon} \in\left(\mathcal{C}, \mathcal{G}_{\alpha, p, n}\right)$ such that for every $\delta \in\left(0, \delta_{\varepsilon}\right)$ and $v \in \operatorname{Lip}_{0}\left(B_{0}(\delta)\right)$,

$$
\|v\|_{L^{\alpha p}\left(B_{0}(\delta), d x\right)} \leq \mathcal{C}_{\varepsilon}\|\nabla v\|_{L^{p}\left(B_{0}(\delta), d x\right)}^{\theta}\|v\|_{L^{\alpha(p-1)+1}\left(B_{0}(\delta), d x\right)}^{1-\theta} .
$$

Let us fix $u \in \operatorname{Lip}_{0}\left(\mathbb{R}^{n}\right)$ arbitrarily and set $v_{\lambda}(x)=\lambda^{\frac{n}{p}} u(\lambda x), \lambda>0$. For $\lambda>0$ large enough, one has $v_{\lambda} \in \operatorname{Lip}_{0}\left(B_{0}(\delta)\right)$. If we plug in $v_{\lambda}$ into (3.2), by using the scaling properties

$\left\|\nabla v_{\lambda}\right\|_{L^{p}\left(B_{0}(\delta), d x\right)}=\lambda\|\nabla u\|_{L^{p}\left(\mathbb{R}^{n}, d x\right)}$ and $\left\|v_{\lambda}\right\|_{L^{q}\left(B_{0}(\delta), d x\right)}=\lambda^{\frac{n}{p}-\frac{n}{q}}\|u\|_{L^{q}\left(\mathbb{R}^{n}, d x\right)}, \quad \forall q>0$,

and the form of the number $\theta$ (see (1.2)), it follows that

$$
\|u\|_{L^{\alpha p}\left(\mathbb{R}^{n}, d x\right)} \leq \mathcal{C}_{\varepsilon}\|\nabla u\|_{L^{p}\left(\mathbb{R}^{n}, d x\right)}^{\theta}\|u\|_{L^{\alpha(p-1)+1}\left(\mathbb{R}^{n}, d x\right)}^{1-\theta} .
$$

\section{定 Springer}


If we insert the extremal function $h_{\alpha, p}^{\lambda}$ of the optimal Gagliardo-Nirenberg inequality on $\mathbb{R}^{n}$ $(\alpha>1)$ into the latter relation, Theorem A yields that $\mathcal{G}_{\alpha, p, n} \leq \mathcal{C}_{\varepsilon}$, a contradiction.

The proofs of (ii) (iii) and (iv) are analogous to (i), taking into account in addition to (3.3) that

$$
\text { Ent }_{d x}\left(\left|v_{\lambda}\right|^{p}\right)=\text { Ent }_{d x}\left(|u|^{p}\right)+n\|u\|_{L^{p}}^{p} \log \lambda,
$$

and

$$
\mathcal{H}^{n}\left(\operatorname{supp}\left(v_{\lambda}\right)\right)=\lambda^{-n} \mathcal{H}^{n}(\operatorname{supp}(u))
$$

respectively.

\subsection{Gagliardo-Nirenberg inequalities on Riemannian manifolds with Ricci $\geq 0$}

Before presenting the proofs of Theorem 1.4 and Corollary 1.1, we recall some results from Munn [17].

To do this, let $(M, g)$ be an $n(\geq 2)$-dimensional complete Riemannian manifold with nonpositive Ricci curvature endowed with its canonical volume element $d v_{g}$. The asymptotic volume growth of $(M, g)$ is defined by

$$
\operatorname{AVG}_{(M, g)}=\lim _{r \rightarrow \infty} \frac{\operatorname{Vol}_{g}\left(B_{x}(r)\right)}{\omega_{n} r^{n}} .
$$

By Bishop-Gromov comparison theorem it follows that $\mathrm{AVG}_{(M, g)} \leq 1$ and this number is independent of the point $x \in M$.

Given $k \in\{1, \ldots, n\}$, let us denote by $\delta_{k, n}>0$ the smallest positive solution to the equation $10^{k+2} C_{k, n}(k) s\left(1+\frac{s}{2 k}\right)^{k}=1$ in variable $s$, where

$$
C_{k, n}(i)= \begin{cases}1 & \text { if } i=0, \\ 3+10 C_{k, n}(i-1)+(16 k)^{n-1}\left(1+10 C_{k, n}(i-1)\right)^{n} & \text { if } i \in\{1, \ldots, k\} .\end{cases}
$$

We now consider the smooth, bijective and increasing function $h_{k, n}:\left(0, \delta_{k, n}\right) \rightarrow(1, \infty)$ defined by

$$
h_{k, n}(s)=\left[1-10^{k+2} C_{k, n}(k) s\left(1+\frac{s}{2 k}\right)^{k}\right]^{-1} .
$$

For every $s>1$, let

$$
\beta(k, s, n)= \begin{cases}1-\left[1+\frac{s^{n}}{\left[h_{1, n}^{-1}(s)\right]^{n}}\right]^{-1} & \text { if } k=1, \\ \max \left\{\beta(1, s, n), \beta\left(i, 1+\frac{h_{k, n}^{-1}(s)}{2 k}, n\right): i=1, \ldots, k-1\right\} & \text { if } k \in\{2, \ldots, n\} .\end{cases}
$$

Note that the constant $\beta(k, s, n)$, which is used to prove the Perelman's maximal volume lemma, denotes the minimum volume growth of $(M, g)$ needed to guarantee that any continuous map $f: \mathbb{S}^{k} \rightarrow B_{x}(\rho)$ has a continuous extension $g: \mathbb{D}^{k+1} \rightarrow B_{x}(c \rho)$, where $\mathbb{D}^{k+1}=\left\{y \in \mathbb{R}^{k+1}:|y| \leq 1\right\}$ and $\mathbb{S}^{k}=\partial \mathbb{D}^{k+1}$, see [17, Definition 3.3]. Finally, the Munn-Perelman constant is defined as

$$
\alpha_{M P}(k, n)=\inf _{s \in(1, \infty)} \beta(k, s, n) .
$$

By construction, $\alpha_{M P}(k, n)$ is non-decreasing in $k$; for numerical values of $\alpha_{M P}(k, n)$ one can consult [17, Appendix A]. 
Proof of Theorem 1.4. Let $(M, g)$ be an $n$-dimensional complete Riemannian manifold with non-negative Ricci curvature $(n \geq 2)$ and assume the $L^{p}$-logarithmic Sobolev inequality $(\mathbf{L S})_{\mathcal{C}}^{p}$ holds on $(M, g)$ for some $p \in(1, n)$ and $\mathcal{C}>0$.

(i) It follows from Lemma 3.1 (iii), i.e., $\mathcal{C} \geq \mathcal{L}_{p, n}$.

(ii) Anderson [2] and $\mathrm{Li}[14]$ stated that if there exists $c_{0}>0$ such that $\operatorname{Vol}_{g}\left(B_{x}(\rho)\right) \geq$ $c_{0} \omega_{n} \rho^{n}$ for every $\rho>0$, then $(M, g)$ has finite fundamental group $\pi_{1}(M)$ and its order is bounded above by $c_{0}{ }^{-1}$. Thus it remains to apply Theorem 1.2.

(iii) Assume that $\mathcal{C}<\alpha_{M P}\left(k_{0}, n\right)^{-\frac{p}{n}} \mathcal{L}_{p, n}$ for some $k_{0} \in\{1, \ldots, n\}$. By Theorem 1.2, we have that

$$
\operatorname{AVG}_{(M, g)}=\lim _{r \rightarrow \infty} \frac{\operatorname{Vol}_{g}\left(B_{x}(r)\right)}{\omega_{n} r^{n}} \geq\left(\frac{\mathcal{L}_{p, n}}{\mathcal{C}}\right)^{\frac{n}{p}}>\alpha_{M P}\left(k_{0}, n\right) \geq \cdots \geq \alpha_{M P}(1, n) .
$$

By Munn [17, Theorem 1.2], it follows that $\pi_{1}(M)=\cdots=\pi_{k_{0}}(M)=0$.

(iv) If $\mathcal{C}<\alpha_{M P}(n, n)^{-\frac{p}{n}} \mathcal{L}_{p, n}$, then $\pi_{1}(M)=\cdots=\pi_{n}(M)=0$, which implies the contractibility of $M$, see e.g. Luft [16].

(v) If $\mathcal{C}=\mathcal{L}_{p, n}$ then by Theorem 1.2 and the Bishop-Gromov volume comparison theorem follows that $\operatorname{Vol}_{g}\left(B_{x}(\rho)\right)=\omega_{n} \rho^{n}$ for every $x \in M$ and $\rho>0$. The equality in Bishop-Gromov theorem implies that $(M, g)$ is isometric to the Euclidean space $\mathbb{R}^{n}$. The converse trivially holds.

Remark 3.1 In the study of heat kernel bounds on an $n$-dimensional complete Riemannian manifold $(M, g)$ with non-negative Ricci curvature, the logarithmic Sobolev inequality

$$
\text { Ent }_{d v_{g}}\left(u^{2}\right) \leq \frac{n}{2} \log \left(C\left\|\nabla_{g} u\right\|_{L^{2}\left(M, d v_{g}\right)}^{2}\right), \quad \forall u \in C_{0}^{\infty}(M),\|u\|_{L^{2}}=1,
$$

plays a central role, $C>0$. In fact, (3.4) is equivalent to an upper bound of the heat kernel $p_{t}(x, y)$ on $M$, i.e.,

$$
\sup _{x, y \in M} p_{t}(x, y) \leq \tilde{C} t^{-\frac{n}{2}}, t>0,
$$

for some $\tilde{C}>0$. According to Theorem B (from Sect. 1.1), the optimal constant in (3.4) in the Euclidean space $\mathbb{R}^{n}$ is given by $C=\mathcal{L}_{n, 2}=\frac{2}{n \pi e}$; this scale invariant form on $\mathbb{R}^{n}$ can be deduced by Gross [10] logarithmic Sobolev inequality

$$
\text { Ent }_{d \gamma_{n}}\left(u^{2}\right) \leq 2\|\nabla u\|_{L^{2}\left(\mathbb{R}^{n}, d \gamma_{n}\right)}^{2}, \quad \forall u \in C_{0}^{\infty}\left(\mathbb{R}^{n}\right),\|u\|_{L^{2}\left(\mathbb{R}^{n}, d \gamma_{n}\right)}=1,
$$

where the canonical Gaussian measure $\gamma_{n}$ has the density $\delta_{n}(x)=(2 \pi)^{-\frac{n}{2}} e^{-\frac{|x|^{2}}{2}}, x \in \mathbb{R}^{n}$, see Weissler [27]. Sharp estimates on the heat kernel shows that on a complete Riemannian manifold $(M, g)$ with non-negative Ricci curvature the $L^{2}$-logarithmic Sobolev inequality (3.4) holds with the optimal Euclidean constant $C=\mathcal{L}_{n, 2}=\frac{2}{n \pi e}$ if and only if $(M, g)$ is isometric to $\mathbb{R}^{n}$, cf. Bakry et al. [4], Ni [18], and Li [14]. In this case, $\tilde{C}=(4 \pi)^{-\frac{n}{2}}$ in (3.5).

In particular, Theorem 1.4 (v) gives a positive answer to the open problem of C. Xia [29] concerning the validity of the optimal $L^{p}$-logarithmic Sobolev inequality for generic $p \in(1, n)$ in the same geometric context as above. Xia's formulation was deeply motivated by the lack of sharp $L^{p}$-estimates $(p \neq 2)$ for the heat kernel on Riemannian manifolds with non-negative Ricci curvature.

\section{定 Springer}


Similar results to Theorem 1.4 can be stated for the other three Gagliardo-Nirenberg-type inequalities; here we formulate one for $(\mathbf{G N 1})_{\mathcal{C}}^{\alpha, p}$, the other two inequalities are left to the reader.

Theorem 3.1 Let $(M, g)$ be an n-dimensional complete Riemannian manifold with nonnegative Ricci curvature $(n \geq 2)$ and assume the $(\mathbf{G N 1})_{\mathcal{C}}^{\alpha, p}$ holds on $(M, g)$ for some $p \in(1, n), \alpha \in\left(1, \frac{n}{n-p}\right]$ and $\mathcal{C}>0$. Then the following assertions hold:

(i) $\mathcal{C} \geq \mathcal{G}_{\alpha, p, n}$;

(ii) The order of the fundamental group $\pi_{1}(M)$ is bounded above by $\left(\frac{\mathcal{C}}{\mathcal{G}_{\alpha, p, n}}\right)^{\frac{n}{\theta}}$;

(iii) If $\mathcal{C}<\alpha_{M P}\left(k_{0}, n\right)^{-\frac{\theta}{n}} \mathcal{G}_{\alpha, p, n}$ for some $k_{0} \in\{1, \ldots, n\}$ then $\pi_{1}(M)=\cdots=\pi_{k_{0}}(M)=$ 0 ;

(iv) If $\mathcal{C}<\alpha_{M P}(n, n)^{-\frac{\theta}{n}} \mathcal{G}_{\alpha, p, n}$ then $M$ is contractible;

(v) $\mathcal{C}=\mathcal{G}_{\alpha, p, n}$ if and only if $(M, g)$ is isometric to the Euclidean space $\mathbb{R}^{n}$.

\subsection{Gagliardo-Nirenberg inequalities on Finsler manifolds with $\boldsymbol{n}$-Ricci $\geq 0$}

Let $M$ be a connected $n$-dimensional $C^{\infty}$-manifold and $T M=\bigcup_{x \in M} T_{x} M$ be its tangent bundle. The pair $(M, F)$ is called a reversible Finsler manifold if a continuous function $F: T M \longrightarrow[0, \infty)$ satisfies the conditions:

(a) $F \in C^{\infty}(T M \backslash\{0\})$;

(b) $F(x, t v)=|t| F(x, v)$ for all $t \in \mathbb{R}$ and $(x, v) \in T M$;

(c) the $n \times n$ matrix $g_{i j}(x, v)=\frac{1}{2} \frac{\partial^{2}\left(F^{2}\right)}{\partial v^{i} \partial v^{j}}(x, v)$ is positive definite for all $(x, v) \in T M \backslash\{0\}$.

Here $v=\sum_{i=1}^{n} v^{i} \frac{\partial}{\partial x^{i}}$, and we shall denote by $g_{v}$ the inner product on $T_{x} M$ induced by the above form. If $g_{i j}(x)=g_{i j}(x, v)$ is independent of $v$ then $(M, F)$ is called Riemannian manifold. A Minkowski space consists of a finite dimensional vector space $V$ and a Minkowski norm which induces a Finsler metric on $V$ by translation, i.e., $F(x, v)$ is independent of $x$. A Finsler manifold $(M, F)$ is called a locally Minkowski space if every point in $M$ admits a local coordinate system $\left(x^{i}\right)$ on its neighborhood such that $F(x, v)$ depends only on $v$ and not on $x$.

We consider on the pull-back bundle $\pi^{*} T M$ the Chern connection, see Bao et al. [5, Theorem 2.4.1]. The coefficients of the Chern connection are denoted by $\Gamma_{j k}^{i}$, which are instead of the well-known Christoffel symbols from Riemannian geometry. A Finsler manifold is of Berwald type if the coefficients $\Gamma_{i j}^{k}(x, v)$ in natural coordinates are independent of $v$. It is clear that Riemannian manifolds and (locally) Minkowski spaces are Berwald spaces. The Chern connection induces in a natural manner on $\pi^{*} T M$ the curvature tensor $R$, see Bao et al. [5, Chapter 3]. By means of the connection, we also have the covariant derivative $D_{v} u$ of a vector field $u$ in the direction $v \in T_{x} M$. Note that $v \mapsto D_{v} u$ is not linear. A vector field $u=u(t)$ along a curve $\sigma$ is parallel if $D_{\dot{\sigma}} u=0$. A $C^{\infty}$ curve $\sigma:[0, a] \rightarrow M$ is a geodesic if $D_{\dot{\sigma}} \dot{\sigma}=0$. Geodesics are considered to be parametrized proportionally to arc-length. The Finsler manifold is complete if every geodesic segment can be extended to $\mathbb{R}$. For a $C^{\infty}$-curve $\sigma:[0, l] \longrightarrow M$, its integral length is given by $L_{F}(\sigma):=\int_{0}^{l} F(\sigma(t), \dot{\sigma}(t)) d t$. Define the distance function $d_{F}: M \times M \longrightarrow[0, \infty)$ by

$$
d_{F}\left(x_{1}, x_{2}\right)=\inf _{\sigma} L_{F}(\sigma),
$$

where $\sigma$ runs over all $C^{\infty}$-curves from $x_{1}$ to $x_{2}$. Geodesics locally minimize $d_{F}$-distances. 
Let $u, v \in T_{x} M$ be two non-collinear vectors and $\mathcal{S}=\operatorname{span}\{u, v\} \subset T_{x} M$. By means of the curvature tensor $R$, the flag curvature of the flag $\{\mathcal{S}, v\}$ is defined by

$$
K(\mathcal{S} ; v)=\frac{g_{v}(R(U, V) V, U)}{g_{v}(V, V) g_{v}(U, U)-g_{v}(U, V)^{2}},
$$

where $U=(v ; u), V=(v ; v) \in \pi^{*} T M$. If $(M, F)$ is Riemannian, the flag curvature reduces to the well known sectional curvature.

Let $v \in T_{x} M$ be such that $F(x, v)=1$ and let $\left\{e_{i}\right\}_{i=1, \ldots, n}$ with $e_{n}=v$ be a basis for $T_{x} M$ such that $\left\{\left(v ; e_{i}\right)\right\}_{i=1, \ldots, n}$ is an orthonormal basis for $\pi_{*} T M$. Let $\mathcal{S}_{i}=\operatorname{span}\left\{e_{i}, v\right\}, i=$ $1, \ldots, n-1$. The Ricci curvature Ric: $T M \rightarrow \mathbb{R}$ is defined by $\operatorname{Ric}(c v)=c^{2} \sum_{i=1}^{n-1} K\left(\mathcal{S}_{i} ; v\right)$ for every $c>0$.

Let $(M, F)$ be an $n$-dimensional complete Finsler manifold and let $\mathrm{m}$ be an arbitrarily positive smooth measure on $M$; such a manifold is viewed as a regular metric measure space and we denote it by $(M, F, \mathrm{~m})$. Let $v \in T_{x} M$ be such that $F(x, v)=1$ and let

$$
\Upsilon(v)=\log \left(\frac{\operatorname{vol}_{g_{v}}(\mathrm{~B}(0,1))}{\mathrm{m}_{x}(\mathrm{~B}(0,1))}\right),
$$

where $\operatorname{vol}_{g_{v}}$ and $\mathrm{m}_{x}$ denote the Lebesgue measures on $T_{x} M$ induced by $g_{v}$ and $\mathrm{m}$, respectively, while $\mathrm{B}(0,1)=\left\{y \in T_{x} M: F(x, y)<1\right\}$ is the unit tangent ball at $T_{x} M$. The latter relation can be rewritten into the more familiar form $\mathrm{m}_{x}(\mathrm{~B}(0,1))=e^{-\Upsilon(v)} \operatorname{vol}_{g_{v}}(\mathrm{~B}(0,1))$. We introduce the notation

$$
\partial_{v} \Upsilon=\left.\frac{d}{d t} \Upsilon(\dot{\sigma}(t))\right|_{t=0},
$$

where $\sigma:(-\varepsilon, \varepsilon) \rightarrow M$ is the geodesic with $\sigma(0)=x$ and $\dot{\sigma}(0)=v$. We say that the space $(M, F, \mathrm{~m})$ has $n$-Ricci curvature bounded below by $K \in \mathbb{R}$ if and only if $\operatorname{Ric}(v) \geq K$ and $\partial_{v} \Upsilon=0$ for every $v \in T_{x} M$ such that $F(x, v)=1$, see Ohta [19, Theorem 1.2] and Ohta and Sturm [21, Definition 5.1]. Note that a Berwald space endowed with the Busemann-Hausdorff measure $\mathrm{m}_{B H}$ (and inducing the volume form $d V_{F}$ ) verifies the property $\partial_{v} \Upsilon \equiv 0$, see Shen [23, Propositions 2.6, 2.7].

The polar transform of $F$ is defined for every $(x, \alpha) \in T^{*} M$ by

$$
F^{*}(x, \alpha)=\sup _{v \in T_{x} M \backslash\{0\}} \frac{\alpha(v)}{F(x, v)} .
$$

Note that, for every $x \in M$, the function $F^{*}(x, \cdot)$ is a Minkowski norm on $T_{x}^{*} M$.

If $u \in \operatorname{Lip}_{0}(M)$, then relation (1.7) can be interpreted as

$$
|\nabla u|_{d_{F}}(x)=F^{*}(x, D u(x)) \text { for a.e. } x \in M,
$$

where $D u(x) \in T_{x}^{*}(M)$ is the distributional derivative of $u$ at $x \in M$, see Ohta and Sturm [21]. In particular, if $(M, F)=(M, g)$ is a Riemannian manifold, then $|\nabla u|_{d_{g}}=\left|\nabla_{g} u\right|$, where $d_{g}$ is the distance function on $(M, g), \nabla_{g}$ is the Riemannian gradient on $(M, g)$, and $|\cdot|$ is the norm coming from the Riemannian metric $g$, respectively.

Although a slightly more general result can be proved, we present an application on Berwald spaces $(M, F)$ endowed with the canonical Busemann-Hausdorff measure $\mathrm{m}_{B H}$ (and its induced volume form $d V_{F}$ ), by exploring the results of Cordero-Erausquin, Nazaret and Villani [6] and Gentil [9] (see Theorems A, B).

Theorem 3.2 [Optimality vs. flatness] Let $(M, F)$ be an n-dimensional complete reversible Berwald space with non-negative Ricci curvature. The following statements are equivalent:

\section{定 Springer}


(i) (GN1) ${ }_{\mathcal{G}_{\alpha, p, n}}^{\alpha, p}$ holds on $(M, F)$ for some $p \in(1, n)$ and $\alpha \in\left(1, \frac{n}{n-p}\right]$;

(ii) (GN2) ${ }_{\mathcal{N}_{\alpha, p, n}}^{\alpha, p}$ holds on $(M, F)$ for some $p \in(1, n)$ and $\alpha \in(0,1)$;

(iii) $(\mathbf{L S})_{\mathcal{L}_{p, n}}^{p}$ holds on $(M, F)$ for some $p \in(1, n)$;

(iv) $(\mathbf{F K})_{\mathcal{F}_{p, n}}^{p}$ holds on $(M, F)$ for some $p \in(1, n)$;

(v) $(M, F)$ is isometric to an n-dimensional Minkowski space.

Proof We divide the proof into two parts.

(i) $\vee($ ii $) \vee($ iii $) \vee($ iv $) \Rightarrow(v)$. Note that the Busemann-Hausdorff measure $\mathrm{m}_{B H}$ satisfies the $n$-density assumption for every $x \in M$, i.e.,

$$
\lim _{\rho \rightarrow 0} \frac{\mathrm{m}_{B H}\left(B_{x}(\rho)\right)}{\omega_{n} \rho^{n}}=1,
$$

see Shen [23, Lemma 5.2]. Since $(M, F)$ is a Berwald space (thus $\partial_{v} \Upsilon \equiv 0$ for every $v \in T_{x} M, x \in M$, see (3.6)), the non-negativity of the Ricci curvature on $(M, F)$ coincides with the non-negativity of the $n$-Ricci curvature on $\left(M, d_{F}, \mathrm{~m}_{B H}\right)$, thus the metric measure space $\left(M, d_{F}, \mathrm{~m}_{B H}\right)$ satisfies the curvature-dimension condition $\mathrm{CD}(0, n)$, see Ohta [19]. Moreover, the completeness of $(M, F)$ via Hopf-Rinow theorem implies that the $\left(M, d_{F}, \mathrm{~m}_{B H}\right)$ is proper. Applying now any of the Theorems 1.1, 1.2 or 1.3 (according to which of the assumptions (i), (ii), (iii) or (iv) is satisfied), it yields that

$$
\mathrm{m}_{B H}\left(B_{x}(\rho)\right) \geq \omega_{n} \rho^{n} \text { for all } x \in M, \rho \geq 0 .
$$

By the generalized Bishop-Gromov theorem on Finsler manifolds and the $n$-density property we also have the reverse inequality, thus

$$
\mathrm{m}_{B H}\left(B_{x}(\rho)\right)=\omega_{n} \rho^{n} \text { for all } x \in M, \rho \geq 0 .
$$

The latter relation immediately implies that the flag curvature on $(M, F)$ is identically zero, see Ohta [19, Theorem 7.3], and Kristály and Ohta [12, Theorem 3.3]. Due to Bao et al. [5, Section 10.5]), every Berwald space with zero flag curvature is necessarily a locally Minkowski space. By (3.9) it follows that $(M, F)$ is actually isometric to a Minkowski space.

$(\mathrm{v}) \Rightarrow(\mathrm{i}) \wedge(\mathrm{ii}) \wedge($ iii $) \wedge($ iv $)$. Let us fix an arbitrary norm $\|\cdot\|$ on $\mathbb{R}^{n}$, and let $\Phi:(M, F) \rightarrow$ $\left(\mathbb{R}^{n},\|\cdot\|\right)$ be an isometry. Then

$$
F(x, y)=\left\|d \Phi_{x}(y)\right\|, x \in M, y \in T_{x} M,
$$

and a simple computation based on the definition of the polar transform (see (3.7)) gives

$$
F^{*}(x, \alpha)=\left\|\alpha d \Phi_{\Phi(x)}^{-1}\right\|_{*}, x \in M, \alpha \in T_{x}^{*} M .
$$

If we consider the change of variables $\tilde{x}=\Phi(x)$, relations (3.8) and (3.10) imply

$$
|\nabla v|_{d_{F}}(x)=F^{*}(x, D v(x))=\left\|\left(D\left(v \circ \Phi^{-1}\right)(\tilde{x})\right)\right\|_{*}, v \in C_{0}^{\infty}(M), x \in M .
$$

Thus, for every $v \in C_{0}^{\infty}(M), p \in(1, n)$ and $q>0$, we have

$$
\begin{aligned}
\left\|D\left(v \circ \Phi^{-1}\right)\right\|_{L^{p}\left(\mathbb{R}^{n}, d \tilde{x}\right)} & =\left(\int_{\mathbb{R}^{n}}\left\|\left(D\left(v \circ \Phi^{-1}\right)(\tilde{x})\right)\right\|_{*}^{p} d \tilde{x}\right)^{\frac{1}{p}}=\left(\int_{M}\left(|\nabla v|_{d_{F}}(x)\right)^{p} d V_{F}(x)\right)^{\frac{1}{p}} \\
& =\left\||\nabla v|_{d_{F}}\right\|_{L^{p}\left(M, d V_{F}\right)},
\end{aligned}
$$

$$
\text { Ent }_{d \tilde{x}}\left(\left|v \circ \Phi^{-1}\right|^{p}\right)=\mathbf{E n t}_{d V_{F}}\left(|v|^{p}\right) \text { and }\left\|v \circ \Phi^{-1}\right\|_{L^{q}}=\|v\|_{L^{q}} \text {. }
$$


It remains to apply the results of Cordero-Erausquin, Nazaret and Villani [6] and Gentil [9] (cf. Theorems A, B) for $u=v \circ \Phi^{-1}$.

Acknowledgments The author is grateful to the Universität Bern for the warm hospitality where this work has been initiated. He thanks Professor Zoltán M. Balogh and Professor Cédric Villani for stimulating conversations on the $L^{p}$-logarithmic Sobolev inequality and Professor Michel Ledoux for suggesting the study of the whole family of Gagliardo-Nirenberg inequalities. Research supported by a Grant of the Romanian National Authority for Scientific Research, CNCS-UEFISCDI, "Symmetries in elliptic problems: Euclidean and nonEuclidean techniques", Project No. PN-II-ID-PCE-2011-3-0241, and by the János Bolyai Research Scholarship of the Hungarian Academy of Sciences.

\section{References}

1. Alvino, A., Ferone, V., Lions, P.L., Trombetti, G.: Convex symmetrization and applications. Ann. Inst. H. Poincaré Anal. Non Lin éaire 14, 275-293 (1997)

2. Anderson, M.: On the topology of complete manifold of nonnegative Ricci curvature. Topology 3, 41-55 (1990)

3. Aubin, T.: Problèmes isopérimétriques et espaces de Sobolev. J. Differ. Geom. 11(4), 573-598 (1976)

4. Bakry, D., Concordet, D., Ledoux, M.: Optimal heat kernel bounds under logarithmic Sobolev inequalities. ESAIM Probab. Stati. 1, 391-407 (1995/97)

5. BaoD, Chern, S.S., Shen, Z.: Introduction to Riemann-Finsler Geometry, Graduate Texts in Mathematics, p. 200. Springer, Berlin (2000)

6. Cordero-Erausquin, D., Nazaret, B., Villani, C.: A mass-transportation approach to sharp Sobolev and Gagliardo-Nirenberg inequalities. Adv. Math. 182(2), 307-332 (2004)

7. Del Pino, M., Dolbeault, J.: The optimal Euclidean $L^{p}$-Sobolev logarithmic inequality. J. Funct. Anal. 197, 151-161 (2003)

8. do Carmo, M.P., Xia, C.: Complete manifolds with non-negative Ricci curvature and the Caffarelli-KohnNirenberg inequalities. Compos. Math. 140, 818-826 (2004)

9. Gentil, I.: The general optimal $L^{p}$-Euclidean logarithmic Sobolev inequality by Hamilton-Jacobi equations. J. Funct. Anal. 202(2), 591-599 (2003)

10. Gross, L.: Logarithmic Sobolev inequalities. Am. J. Math. 97, 1061-1083 (1975)

11. Hebey, E.: Nonlinear analysis on manifolds: Sobolev spaces and inequalities. Courant Lecture Notes in Mathematics, 5. New York University, Courant Institute of Mathematical Sciences, New York; American Mathematical Society, Providence, RI (1999)

12. Kristály, A., Ohta, S.: Caffarelli-Kohn-Nirenberg inequality on metric measure spaces with applications. Math. Ann. 357(2), 711-726 (2013)

13. Ledoux, M.: On manifolds with non-negative Ricci curvature and Sobolev inequalities. Comm. Anal. Geom. 7(2), 347-353 (1999)

14. Li, P.: Large time behavior of the heat equation on complete manifolds with nonnegative Ricci curvature. Ann. Math. 124(1), 1-21 (1986)

15. Lott, J., Villani, C.: Ricci curvature for metric measure spaces via optimal transport. Ann. Math. 169(3), 903-991 (2009)

16. Luft, E.: On contractible open topological manifolds. Invent. Math. 4, 192-201 (1967)

17. Munn, M.: Volume growth and the topology of manifolds with nonnegative Ricci curvature. J. Geom. Anal. 20(3), 723-750 (2010)

18. Ni, L.: The entropy formula for linear heat equation. J. Geom. Anal. 14(1), 87-100 (2004)

19. Ohta, S.: Finsler interpolation inequalities. Calc. Var. Partial Differ. Equ. 36, 211-249 (2009)

20. Ohta, S.: On the measure contraction property of metric measure spaces. Comment. Math. Helv. 82, 805-828 (2007)

21. Ohta, S., Sturm, K.-T.: Heat flow on Finsler manifolds. Commun. Pure Appl. Math. 62(10), 1386-1433 (2009)

22. Perelman, G.: Manifolds of positive Ricci curvature with almost maximal volume. J. Am. Math. Soc. 7, 299-305 (1994)

23. Shen, Z.: Volume comparison and its applications in Riemann-Finsler geometry. Adv. Math. 128, 306-328 (1997)

24. Sturm, K.-T.: On the geometry of metric measure spaces. I. Acta Math. 196(1), 65-131 (2006)

25. Sturm, K.-T.: On the geometry of metric measure spaces. II. Acta Math. 196(1), 133-177 (2006)

26. Talenti, G.: Best constant in Sobolev inequality. Ann. Mat. Pure Appl. 110, 353-372 (1976) 
27. Weissler, F.B.: Logarithmic Sobolev inequalities for the heat-diffusion semigroup. Trans. Am. Math. Soc. 237, 255-269 (1978)

28. Xia, C.: The Gagliardo-Nirenberg inequalities and manifolds of non-negative Ricci curvature. J. Funct. Anal. 224(1), 230-241 (2005)

29. Xia, C.: Sobolev type inequalities on complete Riemannian manifolds. http://www.official.kotaroy.com/ meeting/symposium/abstract.bak/02-xia.pdf 